\title{
Structural and vibrational analyses of new potential anticancer drug 2-(phenylmethyl)-2-azaspiro[5.11] heptadecane-1,3,7-trione
}

\author{
Željko J. Vitnik ${ }^{\text {a, }}{ }^{\text {, J Jelena B. Popović-Đorđević }}{ }^{\mathrm{b}}$, Vesna D. Vitnik ${ }^{\mathrm{a}}$ \\ a Department of Chemistry, Institute of Chemistry, Technology and Metallurgy, University of Belgrade, Studentski trg 12-16, 11001, Belgrade, Serbia \\ ${ }^{\mathrm{b}}$ Department of Chemistry and Biochemistry, Faculty of Agriculture, University of Belgrade, Nemanjina 6, 11080, Belgrade, Serbia
}

\section{A R T I C L E I N F O}

\section{Article history:}

Received 12 December 2016

Received in revised form

1 February 2017

Accepted 2 February 2017

Available online 7 February 2017

Keywords:

2-(Phenylmethyl)-2-azaspiro[5.11]

heptadecane-1,3,7-trione

Vibrational spectra

NMR spectra

NBO analysis

MEP

\begin{abstract}
A B S T R A C T
The establishment of the most stable structures of 2-(phenylmethyl)-2-azaspiro[5.11]heptadecane-1,3,7trione, potential anticancer and antimicrobial drug has been investigated in this work. A detailed interpretation of experimental and calculated IR, UV and NMR spectra were reported. The equilibrium geometry, harmonic vibrational frequencies and electronic properties have been investigated with Density Functional Theory using B3LYP/6-311++G(d,p) method. The scaled theoretical wavenumber showed very good agreement with the experimental values. The charge transfer in the molecule was confirmed with NBO analysis. Ultraviolet-visible spectrum was calculated using TD-DFT method and compared with experimental spectrum. The calculated energy and oscillator strength well reproduce the experimental data. The molecular electrostatic potential surface map portrays potential binding sites of the title molecule.
\end{abstract}

(ㄷ) 2017 Elsevier B.V. All rights reserved.

\section{Introduction}

Cyclic imides, especially five- and six-membered systems, are important group of bioactive molecules. They exhibit antitumor (sesbanimide [1], iso-migrastatin [2], lactimidomycin [3]), antiinflammatory [4], immunomodulatory, antiangiogenic (thalidomide and analogues) [5], and anxiolytic (buspirone [6] and tandospirone [7]) activities.

Isolation and examination of pharmacologicaly active natural glutarimides started in 1960s. It was found that cycloheximide [8] and streptimidone [9-11] act as very potent cytotoxic agents $[12,13]$. Structurally related natural product lactimidomycin (LTM), 12-membered unsaturated macrolide antibiotic, produced by Streptomyces amphibiosporus R310-104 (ATCC 53964), is characterized by a biosynthetically rare glutarimide side chain and display strong in vitro cytotoxicity against a number of human tumor cell lines, in vivo antitumor activity in mice model, and potent antifungal activity [2].

After extensive research controversial drug thalidomide $(\alpha-(N-$ phthalimido)glutarimide) was approved as a drug for treatment of certain cancers (newly diagnosed multiple myeloma) and for

\footnotetext{
* Corresponding author.

E-mail address: zvitnik@chem.bg.ac.rs (Ž.J. Vitnik).
}

complication arrised from leprosy as well as analogues of thalidomide with increased potency, 3-amino-thalidomid (pomalidomid, Pomalyst) and $\alpha$-(3-aminophthalimido)glutarimide (lenalidomid, Revlimid) have been developed [14,15]. Lenalidomid is used for treatment of multiple myeloma, while pomalidomid is recently approved by FDA for treatment of relapsed and refractory multiple myeloma.

In the past decade, antitumor activity in vitro of mitonafide [16], amonafide [17], and naphthalimide [18] derivatives was intensively examined.

In addition, glutarimides have applications in various other fields; for example, they are employed as polymers, activators and stabilizers, vulcanizing agents, lubricant additives, dyes and optical brightening agents, photographic and printing aids [19].

The most typical feature of glutarimide derivatives is that the pharmacological and spectroscopic properties can be readily modified by introduction of substituents in the glutarimide ring. Moreover, we are performing the synthesis and the properties relevant to antiproliferative and antibacterial activities of one series of glutarimide derivatives, with some newly synthesised compounds [20]. Cytotoxicity was tested toward three human cancer cell lines, HeLa, K562 and MDA-MB-453 by MTT assay. Compound 2-(phenylmethyl)-2-azaspiro[5.11] heptadecane-1,3,7-trione (abbreviated as $\mathrm{PAH}$ ), containing 12-membered ketone ring, was 
found to be the most potent toward all tested cell lines $\left(\mathrm{IC}_{50}=9-27 \mu \mathrm{M}\right)$. Also, minimum inhibitory concentration (MIC) determined by broth microdilution method confirmed that compound PAH inhibited the growth of all tested Gram-positive and some of the Gram-negative bacteria.

In this paper we describe our results on 2-(phenylmethyl)-2azaspiro[5.11]heptadecane-1,3,7-trione (PAH) regarding the conformational, vibrational, electronic, NMR and reactivity analyses through spectral measurements. The vibrational spectra of $\mathrm{PAH}$ molecule have been analyzed completely to identify the various normal modes with higher wavenumber accuracy. Density Functional Theory (DFT) B3LYP/6-311++G(d,p) calculations have been performed to support our wavenumber assignments. The natural bond orbital (NBO) analysis has been done to prove the stabilization originating from the hyperconjugation of various intramolecular interactions. The UV-Vis spectroscopic studies along with frontier molecular orbital (FMO) analysis have been used to elucidate charge transfer through the molecule. Additionally, molecular electrostatic potential (MEP) surface is plotted over the optimized geometry to explain the reactivity and potential binding sites of PAH molecule. The calculated results were compared with the experimental and the observed spectra were analyzed in detail.

\section{Experimental details}

Chemicals and solvents (analytical grade) were purchased from Merck (Darmstadt, Germany), Sigma-Aldrich and Fluka. All solvents were dried by standard methods and distilled before use. The sodium hydride was used as $60 \%$ dispersion in mineral oil. The reaction was monitored on silica gel precoated TLC plates, $\mathrm{HF}_{254}$ (Merck, Darmstadt, Germany). PAH was synthesised by tandem process described in our previous paper [21]. The process involved base-catalyzed Michael addition of active methylene compound to secondary acrylamide, followed by intramolecular $\mathrm{N}$-acylation of the carboxamido group. PAH was synthesised in reaction of methyl2-oxocyclododecane carboxylate $(8.32 \mathrm{mmol}), \mathrm{N}$-benzylacrylamide (12.5 mmol), $t$-BuOK (0.47 g, $4.16 \mathrm{mmol})$ and 18-crown-6 ether $(0.3 \mathrm{~g}, 1.25 \mathrm{mmol})$ in a mixture of solvents THF/t-Pentanol (3:1). The reaction mixture was heated $\left(70^{\circ} \mathrm{C}, 3 \mathrm{~h}\right)$ and stirred under argon. Purification by dry-flash chromatography $\left(\mathrm{SiO}_{2}\right.$, hexane/EtOAc) yielded PAH as white crystals in a yield of 55\%. The synthesis of PAH is shown in Scheme S1, Supplementary material.

The chemical structure and purity of the synthesised compound was confirmed by its melting point, ${ }^{1} \mathrm{H}$ and ${ }^{13} \mathrm{C}$ NMR, FT-IR, UV and ESI/HR-MS spectra. FT-IR spectrum was recorded with a Bomem MB 100 spectrophotometer. FT-IR spectrum of this compound was recorded in the region $400-4000 \mathrm{~cm}^{-1}$ at a resolution of $4 \mathrm{~cm}^{-1}$. The ultraviolet absorption spectrum of $\mathrm{PAH}$ was recorded from a $10^{-5}$ molar solution of $\mathrm{PAH}$ in chloroform and in the range 200-500 nm, using Shimadzu $1700 \mathrm{UV} /$ Vis spectrophotometer. ${ }^{1} \mathrm{H}$ and ${ }^{13} \mathrm{C}$ NMR spectra were recorded in $\mathrm{CDCl}_{3}$ using TMS as an internal standard on a Bruker Avance 500 spectrometer at $500 \mathrm{MHz}$ and $125 \mathrm{MHz}$, respectively. ESI-MS spectra were recorded on Agilent Technologies 6210-1210 TOF-LC-ESI-HR/MS instrument.

\section{Computational details}

Initial calculations are shown that molecule of PAH can adopt two envelope conformations of glutarimide ring, two conformers accordingly to position of benzyl group and huge number of conformers for 12-membered ring. For generation of conformers for 12-membered ring the AMMP program [22] and Vega ZZ GUI [23] were used. The conformers are generated using the systematically conformational search and minimized with SP4 force field. Trajectories are analyzed, selected geometries are extracted with
CatDCD, part of VMD program [24], and re-optimized with PM3 and B3LYP methods using Gaussian 09 program package [25]. All generated geometries are minimized without any constraint in the potential energy surface. The stability of the optimized geometries was confirmed by frequency calculations, which gave positive values for all the obtained frequencies. Optimized structural parameters were used in the vibrational frequency, electronic properties and isotropic chemical shift calculations.

The assignments of the calculated wavenumbers were aided by the animation option of Gauss View 5.0 graphical interface from Gaussian programs. Furthermore, the theoretical vibrational spectra of the title compound are interpreted by means of Potential Energy Distribution (PED) using VEDA 4 program [26]. The harmonic frequencies were calculated with B3LYP/6-311++G(d,p) method and then scaled by 0.9604 [27] because the vibrational frequency values computed at this level contain known systematic errors [28]. The nuclear magnetic resonance (NMR) chemical shifts calculations were performed using Gauge- Independent Atomic Orbital (GIAO) method $[29,30]$ at B3LYP level with $6-311++G(d, p)$ basis set and the ${ }^{1} \mathrm{H}$ and ${ }^{13} \mathrm{C}$ isotropic chemical shifts were referenced to the corresponding value for TMS, which was calculated at the same level of theory. The effect of solvent on the theoretical NMR parameters was included using conductor polarizable continuum model (CPCM) [31]. Chloroform $\left(\mathrm{CHCl}_{3}\right)$ was used as solvent.

UV absorption spectrum of this compound was calculated by TD-DFT method in chloroform solvent. The FMO analysis of PAH was also calculated at the same level of theory. The NBO calculations were performed using NBO 3.1 program [32] as implemented in the Gaussian 09 package at the B3LYP/6-311++G(d,p) level. To portray the bonding sites and investigate chemical reactivity of the title molecule, MEP surface, for the 0.002 a.u. isosurfaces of electron density, is plotted over the optimized geometry of the most stable isomer I of PAH using Gauss View 5.0 program.

\section{Results and discussion}

\subsection{Conformational stability}

The investigation of the most stable isomer of PAH, the challenging task in conformational analysis, has been studied. Also, the aim of the conformational analysis of the PAH molecule is to provide the best model for the molecular structure.

Molecule of PAH can adopt four conformers according to glutarimide ring and position of benzyl group and huge number of conformations for 12-membered ring. For each of these four conformers the systematically conformational search was done changing the torsion angles around eleven $\mathrm{C}-\mathrm{C}$ bonds of 12membered ring for $120^{\circ}$ followed by full minimization with SP4 force field. The one of the $\mathrm{C}-\mathrm{C}$ bonds, which contains spiro $\mathrm{C}$ atom, is not included in systematically search to avoid a generation of huge number of unnatural conformers. To retain statistical probability of finding the all relevant conformers, the two searches were done with different $\mathrm{C}-\mathrm{C}$ (spiro) bonds. The all generated trajectories are analyzed and $5 \%$ of energetically most stable geometries of conformers are extracted and re-optimized with PM3 method. All diverse conformers optimized with PM3 which do not differ in energy from the most stable one for more then $10 \mathrm{kcal} / \mathrm{mol}$ were additionally re-optimized with B3LYP/6-31G(d) method. Then the full geometry optimizations of all unique structures were performed by B3LYP method with $6-311++G(d, p)$ basis set.

As a result of these analyses conformer I have been obtained as energetically most stable one and it is shown in Figs. 1 and 2. Fig. 1 also presents the geometries of the fifty stable conformers of PAH (I-L). The energies of these conformers as well as the relative 


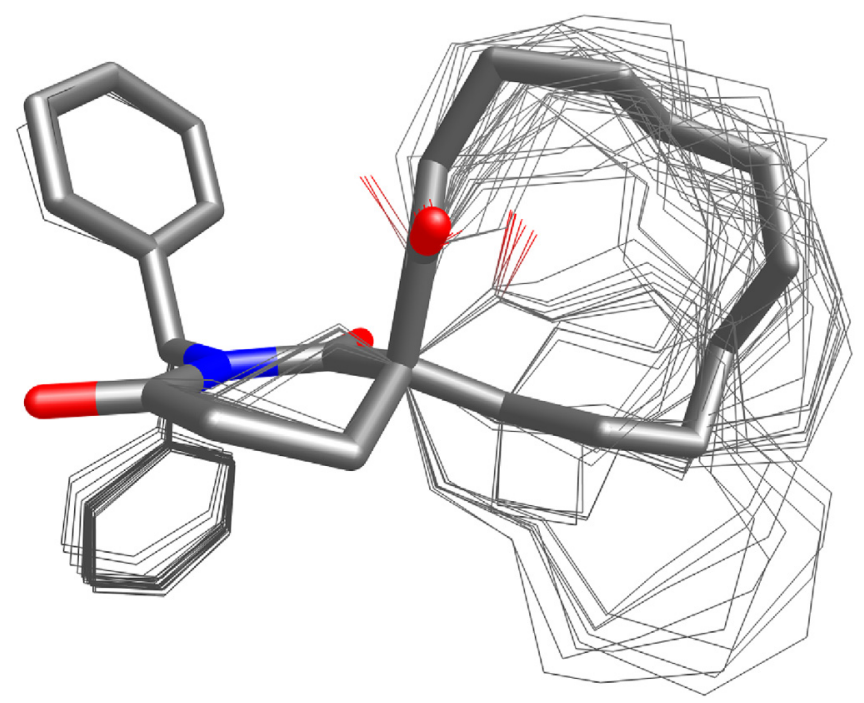

Fig. 1. Superimposed molecular structures of the fifty most stable conformers of 2(phenylmethyl)-2-azaspiro[5.11]heptadecane-1,3,7-trione. The most stable conformer I is presented as tube structure.

energies and statistical Boltzmann distribution weighted values, are presented in Table S1 (Supplementary material). From Fig. 1 it can be seen that conformer I have glutarimide ring in the envelope conformation with $\mathrm{C} 4$ atom under the plane of the glutarimide ring. This position of $\mathrm{C} 4$ atom is a prerequisite for the axial position of $\mathrm{C} 3-\mathrm{C} 7$ bond and at the same time the axial position of $\mathrm{C} 7=\mathrm{O} 10$ carbonyl group of cyclododecanone ring. Also, in this conformation, orientation of $\mathrm{C} 7=010$ group is cis relative to the benzyl group, namely benzyl group is on the upper side of the plane of glutarimide ring. From Table S1 (Supplementary material), it can be concluded that the energy differences between conformers are low and therefore they can easily convert from one to another under the influence of external interactions.

In order to estimate the barrier height and confirm the transitions between conformers for the rotation around the $\mathrm{C}-\mathrm{C}$ and $\mathrm{N}-\mathrm{C}$ bonds potential energy curves are calculated using the B3LYP

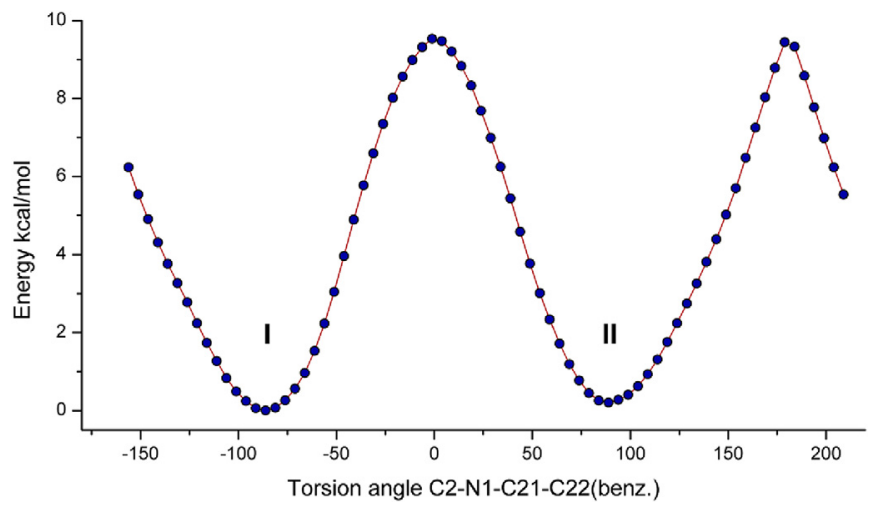

Fig. 3. The potential energy curve of $\mathrm{PAH}$ along the $\mathrm{C} 2-\mathrm{N} 1-\mathrm{C} 21-\mathrm{C} 22$ dihedral angle.

method with $6-31+\mathrm{G}(\mathrm{d})$ basis set. The potential energy surface (PES) study has been carried out to confirm the orientation of benzyl group in PAH molecule. PES for the rotation of the benzyl group about $\mathrm{N} 1-\mathrm{C} 21$ bond was obtained for molecule I by calculating the variation of its total energy with change in the dihedral angle $\tau(\mathrm{C} 2-\mathrm{N} 1-\mathrm{C} 21-\mathrm{C} 22)$ between $0^{\circ}$ and $360^{\circ}$ in intervals of $5^{\circ}$ by B3LYP/6-31 + G(d) calculations. The PES scan for the position of benzyl group of PAH molecule is shown in Fig. 3. For this rotation two minima energy curve have been obtained at -86.13 and $88.83^{\circ}$ (conformers I and II) as shown in Fig. 3 clearly demonstrates that the first minimum corresponds to the most stable conformer I. Thus, in its more stable configuration, the benzyl group is in cis orientation to the carbonyl group of cyclododecanone ring. It should be noted that the energy difference between these two structures is about $0.2 \mathrm{kcal} / \mathrm{mol}$, which confirms that they can easily convert from one to another.

Also, the PES scan for glutarimide part of investigated molecule is shown in Fig. 4. During the calculation all the geometrical parameters were simultaneously relaxed while the dihedral angles C3-C4-C5-C6 were varied form $60^{\circ}$ to $-80^{\circ}$ by steps of $2.5^{\circ}$. Results of the geometry optimizations indicate that the title compound has two envelop conformers XI $\left(-55^{\circ}\right)$ and $\mathbf{I}\left(50^{\circ}\right)$ for investigated dihedral angles, respectively. However, the latter is

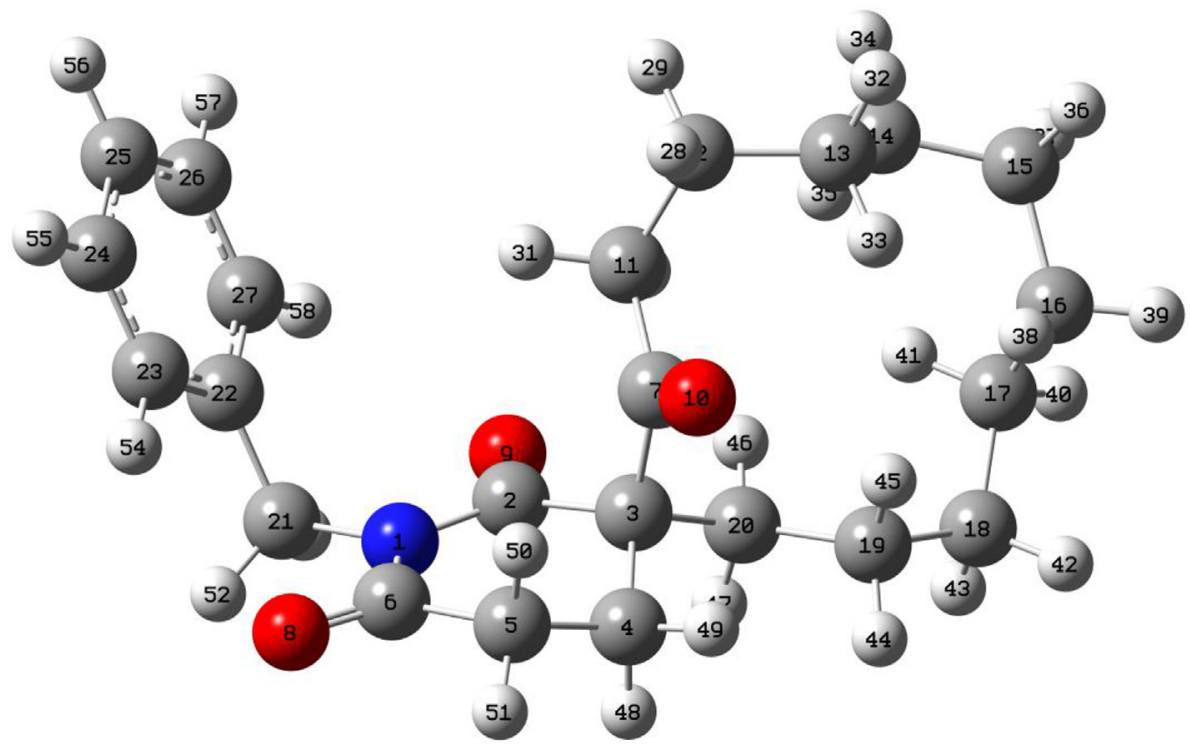

Fig. 2. Optimized molecular structure and atomic numbering of PAH (I). 


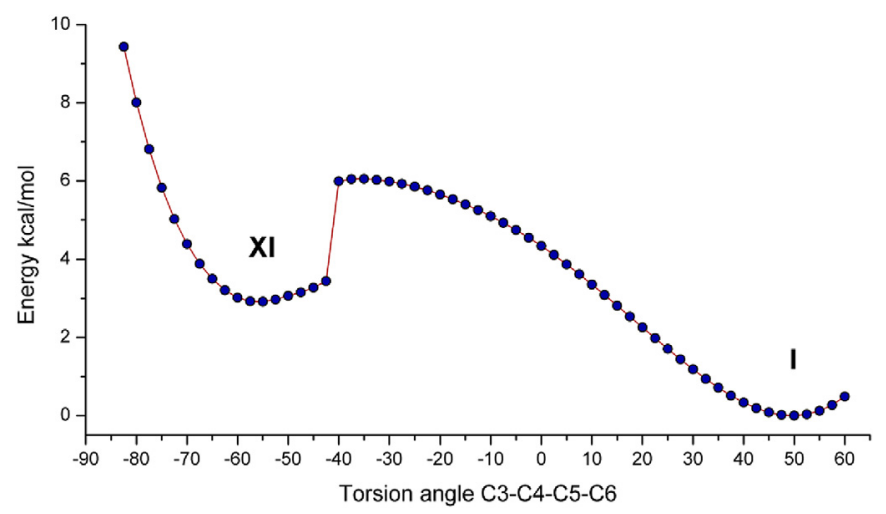

Fig. 4. The potential energy curve of PAH along the $\mathrm{C} 3-\mathrm{C} 4-\mathrm{C} 5-\mathrm{C} 6$ dihedral angles.

deeper than the former and represents the more stable conformation. The potential energy surface diagrams reveal that the conformation with minimum energy corresponds to the structure in which $\mathrm{C} 4$ atom lies under the plane of the glutarimide ring.

The PES scan for the dihedral angle C14-C15-C16-C17 of cyclododecanone part was varied form $0^{\circ}-360^{\circ}$ by steps of $5^{\circ}$. For this rotation, four minima energy curve have been obtained as shown in Fig. 5. The energy difference between these structures is about $5 \mathrm{kcal} / \mathrm{mol}$ and potential energy minimum at $67.4^{\circ}$ corresponds to the most stable conformer $\mathbf{I}$.

\subsection{Molecular geometry}

The optimized geometrical parameters of conformer I of PAH calculated by B3LYP level with $6-311++\mathrm{G}(\mathrm{d}, \mathrm{p})$ basis set are listed in Table S2 (Supplementary material), the atom numbering scheme is given in Fig. 2. There is no X-ray crystal structure for this compound, so the calculated and experimental structural data have not been compared.

\subsection{Vibrational analysis}

The molecule PAH belongs to $\mathrm{C} 1$ point group and possesses 168 normal vibrational modes. The calculated frequencies are slightly higher than the experimental values for almost all of the normal modes. Two factors are responsible for this. The first is caused by the combination of electron correlation effects and basis set deficiencies and the second reason is the fact that the experimental value is an anharmonic frequency while the calculated value is a harmonic frequency. The theoretical harmonic wavenumbers have

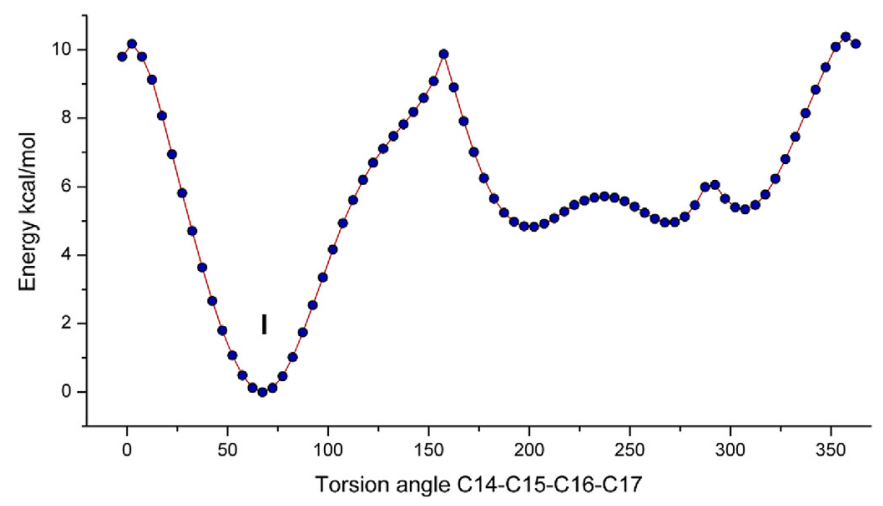

Fig. 5. The potential energy curve of $\mathrm{PAH}$ along the $\mathrm{C} 14-\mathrm{C} 15-\mathrm{C} 16-\mathrm{C} 17$ dihedral angle. been scaled by the scale factor of 0.9604 [27]. After implementing scaling procedure, the theoretically computed wavenumbers matched well with the experimental ones. For comparison, scaled theoretical and experimental FT-IR wavenumbers are presented in Table 1 along with vibrational assignments and calculated IR intensities. The experimental and calculated FT-IR spectra are shown in Fig. 6. The detailed assignments along with the percentage of PED are summarized in Table 1, the assignments having < 10\% (PED percentage) are not presented.

After scaling, correlation graph which presents the experimental (FT-IR) vs. calculated wavenumbers was drawn (Fig. S1, Supplementary material). The relation between these results is linear and described by the equation:

$v_{\text {cal. }}=1.0012 v_{\text {exp. }}-16.0386\left(R^{2}=0.9996\right)$ for B3LYP/6-311 $+\mathrm{G}(\mathrm{d}, \mathrm{p})$

Comparison of the wavenumbers calculated by the DFT/B3LYP method using 6-311++G(d,p) basis set with experimental values confirms that this method show good agreement with correlation coefficient 0.9996, Fig. S1 (Supplementary material).

The investigated molecule consists of a glutarimide ring substituted with a cyclododecanone ring at position 3 and a benzyl group at position 1 . Consequently, the vibrational modes are discussed in tree parts: vibrations of glutarimide ring, vibrations of benzyl group and vibrations of cyclododecanone group.

\subsubsection{Vibrations of glutarimide ring}

4.3.1.1. $C=O$ group vibrations. It is well known in the literature that strongly polar bonds such as carbonyl groups produce very strong bands [33]. On the basis of PED, the prominent absorptions at 1673 and $1725 \mathrm{~cm}^{-1}$ in FT-IR are assigned to $\mathrm{C} 2=09$ and $\mathrm{C} 6=08$ stretching modes, respectively. The results of computations give the wavenumbers of these modes at 1644 and $1699 \mathrm{~cm}^{-1}$. The region below $800 \mathrm{~cm}^{-1}$ is mostly dominated by in-plane and out-ofplane ring vibrations. Also, the $\mathrm{C}-\mathrm{O}$ in- and out-of-plane vibrations are expected in this region. The $\mathrm{C}=\mathrm{O}$ in-plane bending modes are mixed with $\mathrm{C}-\mathrm{N}$ stretching modes and in-plane bending modes of $\mathrm{N}-\mathrm{C}-\mathrm{O}$ group. The bands at 446, 490 and $621 \mathrm{~cm}^{-1}$ in FT-IR are assigned to $\mathrm{C}=\mathrm{O}$ in-plane bending vibrations. The wavenumbers at 439, 486 and $591 \mathrm{~cm}^{-1}$ calculated by B3LYP/6-311++G(d,p) show excellent agreement with the experimental data. The $\mathrm{C}=\mathrm{O}$ out-ofplane vibrations are coupled with out-of-plane ring vibrations and are observed in the region $160-300 \mathrm{~cm}^{-1}$ in calculated IR spectrum.

4.3.1.2. $C-N$ and $C-C$ group vibrations. The identification of $C-N$ vibrations is a difficult task, because of the mixing of bending and stretching bands in region of $1000-1500 \mathrm{~cm}^{-1}$. The $\mathrm{C}-\mathrm{N}$ stretching absorptions assign in the region $1092-1401 \mathrm{~cm}^{-1}$ for substituted piperidines [34]. In 1-(4-Chloro-phenyl)-3-phenyl-succinimide, the $\mathrm{C}-\mathrm{N}$ stretching bands are found to be in the region 1024-1271 $\mathrm{cm}^{-1}$ [35]. In the present work, the FT-IR band observed at $1049,1142,1164$ and $1281 \mathrm{~cm}^{-1}$ are assigned to $\mathrm{C}-\mathrm{N}$ stretching mode. The theoretically calculated values of $\mathrm{C}-\mathrm{N}$ stretching vibrations in the region 1040-1275 $\mathrm{cm}^{-1}$ (mode nos: 91, 84, 82, 69 and 68) coincide with experimental data. The stretching vibration of N1-C21 bond toward benzyl group, assigned at $883 \mathrm{~cm}^{-1}$ (FT-IR) is in agreement with calculated frequency at $877 \mathrm{~cm}^{-1}$ (mode no: 109). The $\mathrm{C}-\mathrm{N}-\mathrm{C}$ in-plane bending vibration assigned at $531 \mathrm{~cm}^{-1}$ (FT-IR) are in agreement with calculated frequencies by B3LYP/6$311++\mathrm{G}(\mathrm{d}, \mathrm{p})$ method at $516 \mathrm{~cm}^{-1}$ (mode no: 132). The $\mathrm{C}-\mathrm{N}-\mathrm{C}$ out-of-plane bending vibration found at $478 \mathrm{~cm}^{-1}$ and correlate well with the computed value at $468 \mathrm{~cm}^{-1}$.

The observed strong bands at 1004 and $1080 \mathrm{~cm}^{-1}$ in FT-IR are assigned to $\mathrm{C}-\mathrm{C}$ stretching modes for glutarimide ring of $\mathrm{PAH}$ 
Table 1

The observed and calculated vibrational frequencies using B3LYP/6-311++G(d,p) method for PAH [harmonic frequency $\left.\left(\mathrm{cm}^{-1}\right), \mathrm{IR}_{\text {int }}\left(\mathrm{km} \mathrm{mol}^{-1}\right)\right]$.

\begin{tabular}{|c|c|c|c|c|c|}
\hline Mode no. & IR $\exp \mathrm{cm}^{-1}$ & Unscaled B3LYP & Scaled B3LYP & IR Inten ${ }^{\mathrm{a}}$ & Assignments $^{\mathrm{b}}, \mathrm{PED}(\%)$ \\
\hline 1 & 3086 & 3191 & 3064 & 9.77 & $v_{\text {sym }} \mathrm{CH}(94)$ \\
\hline 2 & & 3186 & 3060 & 10.94 & $v_{\text {asy }} \mathrm{CH}(93)$ \\
\hline 3 & 3067 & 3180 & 3054 & 14.91 & $v_{\text {asy }} \mathrm{CH}(87)$ \\
\hline 4 & & 3169 & 3043 & 9.47 & $v_{\text {asy }} \mathrm{CH}(98)$ \\
\hline 5 & & 3160 & 3034 & 0.06 & $v_{\text {asy }} \mathrm{CH}(87)+v_{\text {asy }} \mathrm{CH}(11)$ \\
\hline 6 & & 3156 & 3031 & 0.31 & $v_{\text {asy }} \mathrm{C} 21 \mathrm{H}_{2}(100)$ \\
\hline 7 & 3054 & 3116 & 2993 & 9.67 & $v_{\text {asy }} \mathrm{C}_{4} \mathrm{H}_{2}(64)$ \\
\hline 8 & & 3115 & 2992 & 4.31 & $v_{\text {asy }} \mathrm{C}_{4} \mathrm{H}_{2}(81)$ \\
\hline 9 & 3030 & 3106 & 2983 & 15.4 & $v_{\text {asy }} \mathrm{C}_{5} \mathrm{H}_{2}(64)$ \\
\hline 10 & & 3105 & 2982 & 1.82 & $v_{\text {sym }} \mathrm{C} 21 \mathrm{H}_{2}(64)+v_{\text {asy }} \mathrm{C} 5 \mathrm{H}_{2}(17)$ \\
\hline 11 & 3003 & 3104 & 2981 & 14.03 & $v_{\text {sym }} \mathrm{C} 21 \mathrm{H}_{2}(82)+v_{\text {asy }} \mathrm{C} 5 \mathrm{H}_{2}(14)$ \\
\hline 12 & 2981 & 3093 & 2971 & 45.45 & $v_{\text {asy }} \mathrm{CH}_{2}(15)+v_{\text {asy }} \mathrm{CH}_{2}(11)+v_{\text {asy }} \mathrm{CH}_{2}(33)$ \\
\hline 13 & & 3091 & 2968 & 37.31 & $v_{\text {asy }} \mathrm{CH}_{2}(11)+v_{\text {asy }} \mathrm{CH}_{2}(51)$ \\
\hline 14 & 2962 & 3074 & 2952 & 52.88 & $v_{\text {asy }} \mathrm{CH}_{2}(62)$ \\
\hline 15 & 2941 & 3069 & 2947 & 87.73 & $v_{\text {asy }} \mathrm{CH}_{2}(74)$ \\
\hline 16 & & 3060 & 2939 & 32.15 & $v_{\text {asy }} \mathrm{CH}_{2}(62)$ \\
\hline 17 & 2931 & 3056 & 2935 & 9.48 & $v_{\text {sym }} \mathrm{C}^{2} \mathrm{H}_{2}(51)+v_{\mathrm{sym}} \mathrm{C} 4 \mathrm{H}_{2}(19)$ \\
\hline 18 & & 3047 & 2926 & 9.47 & $v_{\mathrm{sym}} \mathrm{CH}_{2}(41)+v_{\mathrm{sym}} \mathrm{CH}_{2}(13)+v_{\mathrm{asy}} \mathrm{CH}_{2}(13)$ \\
\hline 19 & & 3046 & 2925 & 40.06 & $v_{\text {asy }} \mathrm{CH}_{2}(10)+v_{\text {sym }} \mathrm{CH}_{2}(55)$ \\
\hline 20 & & 3037 & 2917 & 9.97 & $v_{\text {sym }} \mathrm{CH}_{2}(79)$ \\
\hline 21 & & 3035 & 2915 & 22.3 & $v_{\text {sym }} \mathrm{C}_{4} \mathrm{H}_{2}(13)+v_{\text {sym }} \mathrm{CH}_{2}(40)$ \\
\hline 22 & & 3033 & 2913 & 33.55 & $v_{\text {sym }} \mathrm{CH}_{2}(77)$ \\
\hline 23 & 2915 & 3033 & 2913 & 34.94 & $v_{\text {sym }} \mathrm{CH}_{2}(75)$ \\
\hline 24 & & 3030 & 2910 & 4.71 & $v_{\text {sym }} \mathrm{C} 4 \mathrm{H} 2(16)+v_{\text {sym }} \mathrm{CH}_{2}(42)$ \\
\hline 25 & & 3028 & 2909 & 22.99 & $v_{\mathrm{sym}} \mathrm{CH}_{2}(31)+v_{\mathrm{sym}} \mathrm{CH}_{2}(39)$ \\
\hline 26 & 2903 & 3027 & 2907 & 29.63 & $v_{\text {sym }} \mathrm{CH}_{2}(61)$ \\
\hline 27 & & 3025 & 2905 & 18.91 & $v_{\text {sym }} \mathrm{CH}_{2}(30)+v_{\text {sym }} \mathrm{CH}_{2}(13)+v_{\mathrm{sym}} \mathrm{CH}_{2}(11)+v_{\text {sym }} \mathrm{CH}_{2}(17)$ \\
\hline 28 & 2866 & 3023 & 2903 & 21.4 & $v_{\text {sym }} \mathrm{CH}_{2}(82)$ \\
\hline 29 & & 3015 & 2895 & 8.39 & $v_{\mathrm{sym}} \mathrm{CH}_{2}(77)$ \\
\hline 30 & & 3004 & 2885 & 28.52 & $v_{\mathrm{sym}} \mathrm{CH}_{2}(83)$ \\
\hline 31 & 2848 & 3003 & 2884 & 33.38 & $v_{\mathrm{sym}} \mathrm{CH}_{2}(83)$ \\
\hline 32 & 1725 & 1769 & 1699 & 149.44 & vOC (74) \\
\hline 33 & 1707 & 1753 & 1684 & 123.5 & vOC (82) \\
\hline 34 & 1673 & 1712 & 1644 & 401.9 & vOC (79) \\
\hline 35 & 1607 & 1644 & 1578 & 2.97 & $v \mathrm{CC}(63)+\beta \mathrm{HCC}(21)$ \\
\hline 36 & 1561 & 1623 & 1559 & 3.56 & $v C C(70)$ \\
\hline 37 & 1525 & 1526 & 1466 & 13.58 & $v \mathrm{CC}(58)+\beta \mathrm{HCC}(28)$ \\
\hline 38 & 1496 & 1522 & 1461 & 25.87 & $\delta \mathrm{CH}_{2}(67)$ \\
\hline 39 & & 1515 & 1455 & 0.46 & $\delta \mathrm{CH}_{2}(59)$ \\
\hline 40 & & 1512 & 1452 & 0.32 & $\delta \mathrm{CH}_{2}(10)+\delta \mathrm{CH}_{2}(55)$ \\
\hline 41 & 1467 & 1507 & 1448 & 1.41 & $\delta \mathrm{C} 4 \mathrm{H}_{2}(67)$ \\
\hline 42 & & 1498 & 1439 & 3.64 & $\delta \mathrm{CH}_{2}(61)+\delta \mathrm{CH}_{2}(10)$ \\
\hline 43 & & 1494 & 1435 & 2.71 & $\delta \mathrm{CH}_{2}(54)$ \\
\hline 44 & & 1492 & 1432 & 9.08 & $\delta \mathrm{CH}_{2}(61)$ \\
\hline 45 & & 1488 & 1430 & 9.41 & $\delta \mathrm{CH}_{2}(71)$ \\
\hline 46 & & 1487 & 1428 & 1.93 & $\delta \mathrm{CH}_{2}(62)$ \\
\hline 47 & & 1486 & 1427 & 2.7 & $\beta \mathrm{HCH}(44)+v \mathrm{CC}(21)$ \\
\hline 48 & 1457 & 1485 & 1426 & 13.12 & $\delta \mathrm{C} \mathrm{H}_{2}(72)$ \\
\hline 49 & & 1484 & 1425 & 3.92 & $\delta \mathrm{CH}_{2}(60)+\delta \mathrm{CH}_{2}(11)$ \\
\hline 50 & 1438 & 1473 & 1414 & 26.53 & $\delta \mathrm{C} 21 \mathrm{H}_{2}(85)$ \\
\hline 51 & 1406 & 1470 & 1411 & 10.26 & $\omega \mathrm{CH}_{2}(83)$ \\
\hline 52 & & 1410 & 1354 & 0.17 & $\omega \mathrm{CH}_{2}(33)$ \\
\hline 53 & & 1407 & 1351 & 1.41 & $\omega \mathrm{CH}_{2}(41)+\tau \mathrm{HCCC}(13)$ \\
\hline 54 & 1382 & 1401 & 1346 & 29.47 & $\omega \mathrm{C} 21 \mathrm{H}_{2}(59)$ \\
\hline 55 & & 1398 & 1343 & 0.99 & $\omega \mathrm{CH}_{2}(11)+\tau \mathrm{HCCC}(42)$ \\
\hline 56 & 1352 & 1390 & 1335 & 31.81 & $\omega\left(\mathrm{C}_{4} \mathrm{H}_{2}+\mathrm{C} 5 \mathrm{H}_{2}\right)(42)$ \\
\hline 57 & & 1387 & 1332 & 13.85 & $\omega \mathrm{CH}_{2}(48)$ \\
\hline 58 & 1320 & 1381 & 1326 & 13.42 & $\beta \mathrm{HCC}(18)+\mathrm{tC} 21 \mathrm{H}_{2}(13)$ \\
\hline 59 & & 1377 & 1323 & 0.5 & $\omega \mathrm{CH}_{2}(29)$ \\
\hline 60 & & 1376 & 1321 & 0.94 & $\beta \mathrm{HCC}(16)+\omega \mathrm{CH}_{2}(22)$ \\
\hline 61 & & 1367 & 1312 & 5.47 & $\omega \mathrm{CH}_{2}(34)$ \\
\hline 62 & & 1364 & 1310 & 6.55 & $\beta \mathrm{HCC}(35)+\mathrm{tC} 4 \mathrm{H}_{2}(14)+\mathrm{tC} 5 \mathrm{H}_{2}(14)$ \\
\hline 63 & 1308 & 1355 & 1302 & 2.76 & $\mathrm{tCH}_{2}(17)$ \\
\hline 64 & & 1353 & 1299 & 24.85 & $v \mathrm{CC}(22)+\beta \mathrm{HCC}(40)$ \\
\hline 65 & 1294 & 1345 & 1292 & 2.91 & $\beta \mathrm{HCC}(30)$ \\
\hline 66 & & 1338 & 1285 & 0.46 & $\beta \mathrm{HCC}(13)+\beta \mathrm{HCC}(18)+\tau \mathrm{HCCC}(11)$ \\
\hline 67 & & 1331 & 1278 & 4.09 & $\beta \mathrm{HCC}(35)$ \\
\hline 68 & & 1328 & 1275 & 156.91 & $v C C(14)+v \mathrm{NC}(14)$ \\
\hline 69 & 1281 & 1326 & 1273 & 105.32 & $v C C(30)+v N C(10)$ \\
\hline 70 & & 1322 & 1269 & 23.85 & $\beta \mathrm{HCC}(10)+\beta \mathrm{HCC}(19)$ \\
\hline 71 & 1259 & 1308 & 1256 & 7.8 & $\beta \mathrm{HCC}(11)$ \\
\hline 72 & & 1303 & 1251 & 4.33 & $\beta \mathrm{HCC}(23)$ \\
\hline 73 & & 1292 & 1241 & 6.81 & $\beta \mathrm{HCC}(19)+\tau \mathrm{HCCC}(10)$ \\
\hline
\end{tabular}


Table 1 (continued)

\begin{tabular}{|c|c|c|c|c|c|}
\hline Mode no. & IR $\exp \mathrm{cm}^{-1}$ & Unscaled B3LYP & Scaled B3LYP & IR Inten $^{\mathrm{a}}$ & Assignments $^{\mathrm{b}}, \mathrm{PED}(\%)$ \\
\hline 74 & 1248 & 1281 & 1231 & 3.36 & $\mathrm{t}\left(\mathrm{C}_{4} \mathrm{H}_{2}+\mathrm{C}_{5} \mathrm{H}_{2}\right)(33)$ \\
\hline 75 & & 1270 & 1219 & 13.22 & $\beta \mathrm{HCC}(18)+\mathrm{tCH}_{2}(12)$ \\
\hline 76 & 1235 & 1236 & 1187 & 3.32 & $\mathrm{tCH}_{2}(28)$ \\
\hline 77 & & 1230 & 1181 & 8.6 & $\beta \mathrm{HCC}(10)$ \\
\hline 78 & & 1221 & 1173 & 5.79 & $v C C(67)+\beta \mathrm{HCC}(17)$ \\
\hline 79 & 1203 & 1213 & 1165 & 13.22 & $\mathrm{tC} 5 \mathrm{H}_{2}(33)$ \\
\hline 80 & 1181 & 1205 & 1157 & 3.22 & $v \mathrm{CC}(20)+\beta \mathrm{HCC}(73)$ \\
\hline 81 & & 1198 & 1150 & 2.64 & $\beta \mathrm{HCH}(28)$ \\
\hline 82 & 1164 & 1185 & 1138 & 73.52 & $v \mathrm{NC}(12)+\beta \mathrm{HCC}(15)$ \\
\hline 83 & & 1182 & 1135 & 2.63 & $\beta \mathrm{HCC}(10)$ \\
\hline 84 & 1142 & 1177 & 1130 & 167.06 & vNC (24) \\
\hline 85 & 1117 & 1152 & 1106 & 2.15 & $\mathrm{tCH}_{2}(10)$ \\
\hline 86 & & 1127 & 1082 & 11.42 & $\tau \operatorname{HCCC}(12)$ \\
\hline 87 & & 1120 & 1076 & 1.85 & $v C C(10)$ \\
\hline 88 & 1080 & 1105 & 1061 & 66.84 & $v C C(13)+\beta \mathrm{HCC}(24)+\beta C C C(18)$ \\
\hline 89 & & 1098 & 1055 & 0.14 & $v C C(60)$ \\
\hline 90 & & 1096 & 1053 & 25.59 & $v C C(10)$ \\
\hline 91 & 1049 & 1083 & 1040 & 11.61 & $v \mathrm{NC}(10)$ \\
\hline 92 & & 1072 & 1030 & 1.5 & vCC (43) \\
\hline 93 & & 1061 & 1019 & 1.75 & $v C C(10)$ \\
\hline 94 & 1031 & 1056 & 1014 & 25.04 & $v C C(12)$ \\
\hline 95 & 1013 & 1051 & 1009 & 8.68 & $\beta C C C(38)+v C C(14)+\beta \operatorname{HCC}(16)+\beta C C C$ (18) \\
\hline 96 & & 1046 & 1005 & 2.56 & vCC $(22)$ \\
\hline 97 & & 1035 & 994 & 2.6 & $v C C(44)$ \\
\hline 98 & 1004 & 1024 & 984 & 10.98 & $v C C(12)$ \\
\hline 99 & & 1018 & 978 & 0.08 & $v C C(42)+\beta C C C(45)$ \\
\hline 100 & & 1016 & 975 & 3.97 & $v C C(36)$ \\
\hline 101 & & 1006 & 966 & 2.6 & $\gamma \mathrm{HCC}(73)$ \\
\hline 102 & 966 & 1003 & 963 & 52.76 & vCC (19) \\
\hline 103 & & 994 & 955 & 0.98 & $\gamma \mathrm{HCC}(78)$ \\
\hline 104 & 955 & 986 & 947 & 3.08 & $\rho_{C H}(10)$ \\
\hline 105 & 942 & 965 & 927 & 15.31 & $\rho_{\mathrm{CH}}(10)$ \\
\hline 106 & 927 & 951 & 913 & 14.02 & $\gamma \mathrm{HCC}(15)+\tau \mathrm{HCNC}$ \\
\hline 107 & & 939 & 901 & 7.55 & $v C C(11)$ \\
\hline 108 & & 926 & 890 & 1.02 & $v C C(10)$ \\
\hline 109 & 883 & 914 & 877 & 2.22 & $v \mathrm{NC}(11)$ \\
\hline 110 & 863 & 880 & 845 & 1.55 & $\rho C 5 \mathrm{H}_{2}(16)$ \\
\hline 111 & 849 & 877 & 842 & 6.12 & $\rho\left(C 4 \mathrm{H}_{2}+\mathrm{C} 21 \mathrm{H}_{2}\right)(10)$ \\
\hline 112 & & 868 & 834 & 1.99 & vCC (38) \\
\hline 113 & 835 & 864 & 830 & 0.78 & $\gamma \mathrm{HCC}(97)$ \\
\hline 114 & & 839 & 806 & 7.46 & $\rho \mathrm{CH}_{2}(10)$ \\
\hline 115 & 819 & 838 & 805 & 2.13 & $v C C(11)+v C C(17)+\beta C C N(15)+\tau \operatorname{HCCC}(16)$ \\
\hline 116 & & 815 & 783 & 5.1 & $v C C(16)$ \\
\hline 117 & 804 & 807 & 775 & 1.33 & $\rho \mathrm{CH}_{2}(27)$ \\
\hline 118 & & 795 & 763 & 1.85 & vCC $(22)$ \\
\hline 119 & 744 & 772 & 742 & 13.61 & $\gamma \mathrm{HCC}(41)$ \\
\hline 120 & 731 & 765 & 735 & 1.55 & rOCNC (33) \\
\hline 121 & & 742 & 713 & 3.27 & $\tau \mathrm{HCCC}(12)+\tau \mathrm{HCCC}(10)$ \\
\hline 122 & 708 & 731 & 702 & 18.06 & $\rho \mathrm{CH}_{2}(10)+\rho \mathrm{CH}_{2}(32)$ \\
\hline 123 & & 714 & 686 & 52.16 & $\tau \mathrm{HCCC}(10)+\tau \mathrm{HCCC}(31)+\tau \mathrm{HCCC}(35)$ \\
\hline 124 & & 710 & 682 & 12.31 & $\beta C C C(10)+\gamma \mathrm{OCCC}(24)$ \\
\hline 125 & & 704 & 676 & 4.29 & $\tau \mathrm{HCCC}(53)$ \\
\hline 126 & 662 & 677 & 651 & 18.98 & $\gamma \mathrm{OCCC}(26)$ \\
\hline 127 & 637 & 639 & 614 & 13.59 & $\beta C C N(10)+\gamma \mathrm{OCCC}(14)$ \\
\hline 128 & & 636 & 611 & 0.11 & $\beta C C C(85)$ \\
\hline 129 & 621 & 616 & 591 & 7.06 & $v \mathrm{NC}(10)+\beta \mathrm{CCO}(10)$ \\
\hline 130 & & 610 & 586 & 17.04 & $\beta C C O(23)$ \\
\hline 131 & 553 & 566 & 543 & 3.8 & $\tau \mathrm{HCCN}(16)+\gamma \mathrm{OCCC}(17)$ \\
\hline 132 & 531 & 537 & 516 & 3.79 & $v \mathrm{NC}(16)+\beta C \mathrm{CO}(16)+\beta \mathrm{CNC}$ (15) \\
\hline 133 & & 523 & 503 & 1.91 & $\beta C C C(12)$ \\
\hline 134 & 490 & 506 & 486 & 16.83 & $\beta C C O(21)+\beta C C C(12)$ \\
\hline 135 & 478 & 488 & 468 & 10.36 & $\gamma \mathrm{CCC}(12)+\gamma \mathrm{CNC}(15)+\tau \mathrm{CNCC}$ \\
\hline 136 & 465 & 471 & 452 & 3.25 & $\gamma \mathrm{CCO}(13)$ \\
\hline 137 & 446 & 457 & 439 & 5.04 & $v \mathrm{NC}(10)+\beta \mathrm{CCO}(23)+\beta \mathrm{NCO}(11)$ \\
\hline 138 & 427 & 430 & 413 & 14.2 & $\beta C N C(44)+\tau$ HCCC (12) \\
\hline 139 & & 416 & 399 & 3.51 & $\beta C C C(21)$ \\
\hline 140 & & 415 & 399 & 0.51 & $\gamma \operatorname{CCC}(16)+\tau \operatorname{CCCC}(71)$ \\
\hline 141 & & 405 & 389 & 13.3 & $\beta C C C(13)+\beta C C O(10)+\beta C N C$ (13) \\
\hline 142 & & 382 & 367 & 0.35 & $\beta C C C(11)+\tau \operatorname{CCCC}(11)$ \\
\hline 143 & & 365 & 350 & 0.17 & $\beta C C C(11)+\beta C C C(15)$ \\
\hline 144 & & 339 & 326 & 0.47 & $\gamma \operatorname{CCCC}(10)$ \\
\hline 145 & & 316 & 303 & 4.12 & $\gamma \operatorname{CCCO}(16)$ \\
\hline 146 & & 300 & 288 & 6.32 & $\gamma \mathrm{CNC}(12)$ \\
\hline 147 & & 282 & 271 & 0.19 & $\tau \mathrm{CCCC}(11)$ \\
\hline 148 & & 278 & 267 & 1.22 & $\gamma \operatorname{CCCC}(12)$ \\
\hline
\end{tabular}


Table 1 (continued)

\begin{tabular}{|c|c|c|c|c|c|}
\hline Mode no. & IR $\exp \mathrm{cm}^{-1}$ & Unscaled B3LYP & Scaled B3LYP & IR Inten ${ }^{a}$ & Assignments ${ }^{\mathrm{b}}, \mathrm{PED}(\%)$ \\
\hline 149 & & 268 & 257 & 1.29 & $\beta C C C(21)+\tau \operatorname{HCCC}(13)+\tau \operatorname{CCCC}(22)$ \\
\hline 150 & & 254 & 244 & 0.5 & $v C C(24)+\beta C C C(25)$ \\
\hline 151 & & 250 & 240 & 1.57 & $\beta C C C(34)$ \\
\hline 152 & & 226 & 217 & 0.1 & $\beta C C C(36)+\beta C C C(11)$ \\
\hline 153 & & 216 & 208 & 0.76 & $\beta C C C(20)$ \\
\hline 154 & & 198 & 191 & 0.68 & $\gamma \mathrm{CNC}(25)$ \\
\hline 155 & & 184 & 177 & 2.76 & $\tau \mathrm{HCCC}(15)+\tau \mathrm{CCCN}(12)$ \\
\hline 156 & & 183 & 175 & 0.43 & $\tau \operatorname{CCCC}(24)$ \\
\hline 157 & & 177 & 170 & 1.63 & $\beta C C C(10)+\beta C C C(11)+\beta C C C(12)$ \\
\hline 158 & & 160 & 154 & 5.34 & $\tau \mathrm{CCCC}(15)+\tau \mathrm{CNCC}(11)$ \\
\hline 159 & & 134 & 129 & 2.64 & $\tau \operatorname{CCCC}(31)$ \\
\hline 160 & & 109 & 105 & 4.08 & $\tau \mathrm{CCCC}(14)+\tau \mathrm{CCCN}(13)$ \\
\hline 161 & & 95 & 91 & 0.92 & $\tau \operatorname{CCCC}(26)$ \\
\hline 162 & & 82 & 79 & 1.83 & $\tau \mathrm{CCCC}(11)$ \\
\hline 163 & & 73 & 70 & 0.33 & $\beta \operatorname{CCC}(13)+\tau \operatorname{CCCC}(19)$ \\
\hline 164 & & 62 & 59 & 0.36 & $\gamma \operatorname{CCCC}(26)$ \\
\hline 165 & & 41 & 40 & 0.49 & $\beta C C N(11)+\tau \operatorname{CCCN}(11)+\tau \operatorname{CNCC}(20)$ \\
\hline 166 & & 34 & 32 & 0.29 & $\gamma \operatorname{CCCN}(51)$ \\
\hline 167 & & 26 & 25 & 0.04 & $\gamma \mathrm{CCCN}(65)$ \\
\hline 168 & & 16 & 15 & 0.06 & $\tau \mathrm{CNCC}(66)$ \\
\hline
\end{tabular}

${ }^{\mathrm{a}} \mathrm{IR}_{\mathrm{Int}}$ - IR intensity; $\mathrm{km} \mathrm{mol}^{-1}$.

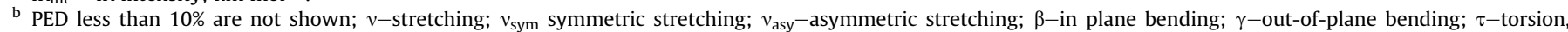
$\omega$-wagging; t-twisting; $\delta$-scissoring; $\rho$-rocking.
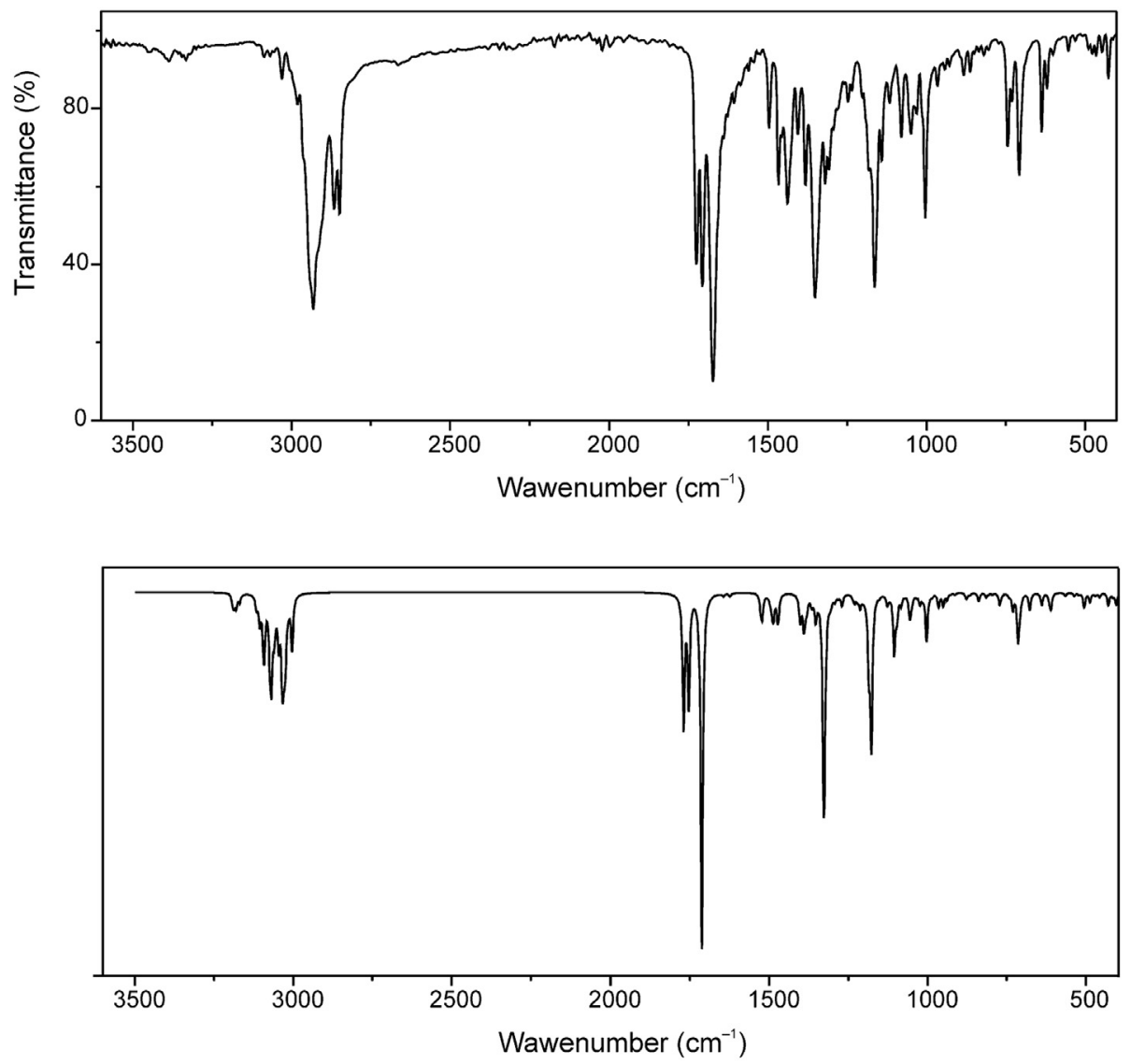

Fig. 6. Comparison of experimental (top) and scaled theoretical IR spectra of PAH.

molecule. The wavenumbers at 984 and $1061 \mathrm{~cm}^{-1}$ (mode nos: 98 and 88 ) calculated by B3LYP/6-311++G(d,p) show excellent agreement with the experimental data. Almost the $\mathrm{C}-\mathrm{C}$ stretching vibrations are found overlapped with other vibrations and difficult to be assigned. Thus the $\mathrm{C}-\mathrm{C}$ stretching mode 88 is coupled with inplane bending $\mathrm{C}-\mathrm{H}$ and $\mathrm{C}-\mathrm{C}$ vibrations. However, the mode 97 is pure mode and the PED contribution to this mode is $44 \%$. The $\mathrm{C}-\mathrm{C}-\mathrm{C}$ bending modes are coupled with in-plane bending $\mathrm{C}-\mathrm{C}-\mathrm{O}$ vibrations for glutarimide ring. In FT-IR, band at $490 \mathrm{~cm}^{-1}$ is designated as $\mathrm{C}-\mathrm{C}-\mathrm{C}$ in-plane bending modes. The calculated counterparts belong to $\mathrm{C}-\mathrm{C}-\mathrm{C}$ in-plane vibrations are observed in the region $486-611 \mathrm{~cm}^{-1}$. In the present work, the calculated 
theoretical wavenumbers 267 and $326 \mathrm{~cm}^{-1}$ depicted in Table 1 are assigned as $\mathrm{C}-\mathrm{C}-\mathrm{C}$ out-of-plane vibrations.

4.3.1.3. $\mathrm{C}_{4} \mathrm{H}_{2}$ and $\mathrm{C}_{5} \mathrm{H}_{2}$ group vibrations. The $\mathrm{C}-\mathrm{H}$ stretching vibrations of the methylene group are at lower frequencies than those of the aromatic $\mathrm{C}-\mathrm{H}$ ring stretching. The asymmetric $\mathrm{CH}_{2}$ stretching vibrations generally observed in the region is $3000-2900 \mathrm{~cm}^{-1}$, while the $\mathrm{CH}_{2}$ symmetric stretching vibrations are between 2900 and $2800 \mathrm{~cm}^{-1}$ [34].

The $\mathrm{CH}_{2}$ asymmetric and symmetric stretching vibrations of $\mathrm{C}_{4} \mathrm{H}_{2}$ and $\mathrm{C}_{5} \mathrm{H}_{2}$ group are observed in FT-IR spectrum as weak intensity bands as seen in Table 1 for our title molecule. The band at $2931 \mathrm{~cm}^{-1}$ in FT-IR spectrum is assigned to $\mathrm{CH}_{2}$ symmetric stretching vibrations, for both $\mathrm{C}_{4} \mathrm{H}_{2}$ and $\mathrm{C}_{5} \mathrm{H}_{2}$ groups. The theoretically computed wavenumbers for these groups are in the range 2910-2935 $\mathrm{cm}^{-1}$ (mode nos. 24, 21 and 17). The weak intensity bands at 3030 and $3054 \mathrm{~cm}^{-1}$ in FT-IR spectrum are attributed to $\mathrm{CH}_{2}$ asymmetric stretching vibrations, for $\mathrm{C}_{5} \mathrm{H}_{2}$ and $\mathrm{C}_{4} \mathrm{H}_{2}$ group, respectively, as shown in Table 1 and correlate well with the calculated values at 2983 and $2993 \mathrm{~cm}^{-1}$, for $\mathrm{C}_{5} \mathrm{H}_{2}$ and $\mathrm{C}_{4} \mathrm{H}_{2}$ group, respectively.

The fundamental bending $\mathrm{CH}_{2}$ vibrations of PAH appear in the frequency region $800-1500 \mathrm{~cm}^{-1}$. These vibrations revealed to be mixed with $\mathrm{C}-\mathrm{C}$ and $\mathrm{C}-\mathrm{N}$ stretching as well as with $\mathrm{C}-\mathrm{C}-\mathrm{C}$ bending vibrations of glutarimide ring. In FT-IR spectrum of PAH, the weak bands at $1438-1496 \mathrm{~cm}^{-1}$ assigned to scissoring vibrations of $\mathrm{CH}_{2}$ groups. The theoretical wavenumber of $\mathrm{CH}_{2}$ scissoring vibrations 1426 and $1448 \mathrm{~cm}^{-1}$ (mode nos: 48 and 41) coincide very well with experimental values at 1457 and $1467 \mathrm{~cm}^{-1}$ for $\mathrm{C}_{5} \mathrm{H}_{2}$ and $\mathrm{C}_{4} \mathrm{H}_{2}$ unit, respectively. The FT-IR wagging mode at $1352 \mathrm{~cm}^{-1}$ corresponding to $\mathrm{C}_{5} \mathrm{H}_{2}$ and $\mathrm{C}_{4} \mathrm{H}_{2}$ units was calculated to be at $1335 \mathrm{~cm}^{-1}$ (mode no: 56). In the present work, the FT-IR frequencies observed in the range $1203-1248 \mathrm{~cm}^{-1}$ have been assigned to $\mathrm{CH}_{2}$ twisting vibrations. The theoretically computed values in the range $1165-1231 \mathrm{~cm}^{-1}$ (mode nos: 79,76 and 74 ) show excellent agreement with experimental data. $\mathrm{The}^{\mathrm{C}} \mathrm{H}_{2}$ rocking vibrations calculated to be 842 and $845 \mathrm{~cm}^{-1}$ (mode nos: 111 and 110) are also in excellent agreement with recorded values at 849 and $863 \mathrm{~cm}^{-1}$.

\subsubsection{Vibrations of benzyl group}

Vibrations of benzyl group are composed of phenyl group and $\mathrm{C}_{2} 1 \mathrm{H}_{2}$ group vibrations.

4.3.2.1. Vibrations of phenyl group. Aromatic $\mathrm{C}-\mathrm{H}$ stretching vibrations generally occur in the region $3000-3100 \mathrm{~cm}^{-1}$ [35]. The theoretical description for this region is somewhat difficult due to the weakness of $\mathrm{C}-\mathrm{H}$ stretching modes. For $\mathrm{PAH}$, the $\mathrm{C}-\mathrm{H}$ stretching aromatic ring vibrations predicted at $3034-3064 \mathrm{~cm}^{-1}$ for B3LYP/6-31++G(d,p) level of theory. These vibrations observed experimentally at $3067-3086 \mathrm{~cm}^{-1}$ in the FT-IR spectrum for PAH. The asymmetric stretching vibration of phenyl group is observed as low intense band at $3067 \mathrm{~cm}^{-1}$, while the symmetric stretching vibration is observed at $3086 \mathrm{~cm}^{-1}$ in the experimental spectra. The scaled theoretical values at B3LYP/6-311++G(d,p) of aromatic ring $\mathrm{C}-\mathrm{H}$ stretching modes coincide well with that of experimental data as depicted in Table 1 . The percentage of PED predicts that $\mathrm{C}-\mathrm{H}$ modes of PAH are very pure. The benzene ring $\mathrm{C}-\mathrm{H}$ in-plane bending vibrations are usually medium and observed in the region $1035-1558 \mathrm{~cm}^{-1}$, while the $\mathrm{C}-\mathrm{H}$ out-of-plane bending vibrations lie in the region $702-928 \mathrm{~cm}^{-1}$ [35]. In-plane $\mathrm{C}-\mathrm{H}$ bending vibrations of phenyl group are observed in the range of 1164-1607 $\mathrm{cm}^{-1}$ and out-of-plane bending vibrations at $744-927 \mathrm{~cm}^{-1}$ region, for PAH in the FT-IR spectrum. The in-plane $\mathrm{C}-\mathrm{H}$ bending vibrations of phenyl group are observed as medium intense bands at 1164, 1181, 1320, 1525 and $1607 \mathrm{~cm}^{-1}$ in FT-IR and the corresponding calculated value are 1138, 1157, 1326, 1466 and $1578 \mathrm{~cm}^{-1}$. In-plane $\mathrm{C}-\mathrm{H}$ bending vibration are also observed at 1173 and $1299 \mathrm{~cm}^{-1}$ in calculated IR spectrum which are mixed with stretching $\mathrm{C}-\mathrm{C}$ vibrations of phenyl group. The weak IR bands at 744,835 and $927 \mathrm{~cm}^{-1}$ are identified as $\mathrm{C}-\mathrm{H}$ out-plane bends of phenyl ring and the corresponding calculated values are 742,830 and $913 \mathrm{~cm}^{-1}$. The theoretical wavenumbers of $\mathrm{C}-\mathrm{H}$ in-plane and out-of-plane are well supported with those of the experimental ones.

The phenyl ring $\mathrm{C}-\mathrm{C}$ stretching vibrations usually occur in the region $1421-1625 \mathrm{~cm}^{-1}$ [36]. The assignment of the skeletal $\mathrm{C}-\mathrm{C}$ stretching modes in FT-IR is quite difficult, since these bands are masked by the more intense bands at $1200-1600 \mathrm{~cm}^{-1}$ originating from the $\mathrm{CH}_{2}$ deformation vibrations. In the present work, phenyl ring $\mathrm{C}-\mathrm{C}$ stretching vibrations are observed at $1281,1525,1561$, and $1607 \mathrm{~cm}^{-1}$, in accordance with the computed values at 1273,1466 , 1559 and $1578 \mathrm{~cm}^{-1}$ (mode nos: 69, 37, 36 and 35), respectively. In the present study, the bands observed at 1013 and $1080 \mathrm{~cm}^{-1}$ in FTIR are assigned to ring in-plane bending modes for phenyl group, and the corresponding calculated values are 1009 and $1061 \mathrm{~cm}^{-1}$ (mode nos: 95 and 88). The ring out-of-plane bending mode wavenumber is observed at $478 \mathrm{~cm}^{-1}$ in FT-IR spectrum and correlate well with the corresponding calculated value at $468 \mathrm{~cm}^{-1}$.

4.3.2.2. $\mathrm{C}_{21 \mathrm{H}_{2}}$ group vibrations. The methylene group $\mathrm{C}_{2} 1 \mathrm{H}_{2}$ have two stretching and the couple of scissoring, wagging, rocking and twisting modes. The symmetric stretching band of this group is observed at $3003 \mathrm{~cm}^{-1}$ and correlated well with the theoretically scaled frequencies at 2981 and $2982 \mathrm{~cm}^{-1}$. The asymmetric stretching band of $\mathrm{C}_{21 \mathrm{H}_{2}}$ group is calculated at $3031 \mathrm{~cm}^{-1}$. The $\mathrm{CH}_{2}$ scissoring mode generates band at $1438 \mathrm{~cm}^{-1}$ in FT-IR is matched well with predicted value at $1414 \mathrm{~cm}^{-1}$. The medium strong band at $1382 \mathrm{~cm}^{-1}$ in FT-IR is attributed to $\mathrm{CH}_{2}$ wagging and correlate well with calculated value at $1346 \mathrm{~cm}^{-1}$. For $\mathrm{C}_{21 \mathrm{H}_{2}}$ group of the compound, the peaks at 849 and $1320 \mathrm{~cm}^{-1}$ in FT-IR are ascribed to rocking and twisting vibrations and the corresponding calculated values are 842 and $1326 \mathrm{~cm}^{-1}$, respectively.

\subsubsection{Vibrations of cyclododecanone group}

4.3.3.1. $C 7=010$ group vibrations. The carbonyl stretching wavenumber of $\mathrm{C} 7=\mathrm{O} 10$ group of cyclododecanone ring of $\mathrm{PAH}$ is observed at $1707 \mathrm{~cm}^{-1}$. The results of computations give the wavenumber of this mode at $1684 \mathrm{~cm}^{-1}$ (mode no. 33). Compared with the $\mathrm{C} 6=\mathrm{O} 8$ group, this group has lower stretching frequency (shift for $18 \mathrm{~cm}^{-1}$ to lower wavenumber), but it is underestimated to the stretching frequency of $\mathrm{C} 2=09$. It is well known from the literature that the most intensive peak in the spectrum of cyclododecanone is the $\mathrm{C}=\mathrm{O}$ stretching which is observed at $1713 \mathrm{~cm}^{-1}$ [37]. Also, the very strong intense bands observed in the region $1711-1715 \mathrm{~cm}^{-1}$ can be assigned to $\mathrm{C}=\mathrm{O}$ stretching vibrations in FT-IR spectra of derivatives of cyclododecanone [38]. The vibrational spectrum shows a medium intensive band around the $600 \mathrm{~cm}^{-1}$. The $\mathrm{C7}-010$ in-plane deformations are observed at 490 , 531 and $621 \mathrm{~cm}^{-1}$, respectively and these results are in according with calculated values at 486,516 and $591 \mathrm{~cm}^{-1}$. The out-of-plane deformation of this carbonyl group is observed at $465 \mathrm{~cm}^{-1}$ and calculated value is $452 \mathrm{~cm}^{-1}$.

4.3.3.2. $\mathrm{CH}_{2}$ group vibrations. As can be seen from Table 1 , there are two asymmetric and four symmetric $\mathrm{CH}_{2}$ stretching vibrations of aliphatic $\mathrm{CH}_{2}$ groups of cyclododecanone moiety. The asymmetric stretching bands of methylene hydrogens $\left(\mathrm{CH}_{2}\right)$ are observed in region $2941-2981 \mathrm{~cm}^{-1}$. The corresponding calculated values are in the region $2939-2971 \mathrm{~cm}^{-1}$. The symmetric stretching bands of 
methylene hydrogens are observed at 2848, 2866, 2903 and $2915 \mathrm{~cm}^{-1}$ and correlate with the calculated values in the region 2884-2926 $\mathrm{cm}^{-1}$. The fundamental $\mathrm{CH}_{2}$ vibrations are able to scissoring, wagging, twisting and rocking appear in the frequency region $700-1400 \mathrm{~cm}^{-1}$ [39]. In title molecule, the scaled vibrational frequencies in the range of $1461-1428 \mathrm{~cm}^{-1}$ (mode nos. 38-46) are assigned to scissoring modes of methylene groups of cyclododecanone moiety are in good agreement with experimental value at $1496 \mathrm{~cm}^{-1}$. The $\mathrm{CH}_{2}$ wagging mode generate band $1406 \mathrm{~cm}^{-1}$ and the scaled calculated wagging modes are in the range $1332-1411 \mathrm{~cm}^{-1}$, Table 1 . Methylene twisting vibration $\left(\mathrm{tCH}_{2}\right)$ of cyclododecanone ring has medium strong peak at $1235 \mathrm{~cm}^{-1}$ in FT-IR and is consistent with calculated values in the range $1187-1219 \mathrm{~cm}^{-1}$. Four bands assigned to the rocking vibrations of methylene units are observed at 708, 804, 942 and $955 \mathrm{~cm}^{-1}$ and the calculated value are 702, 775, 927 and $947 \mathrm{~cm}^{-1}$.

\subsection{4. $C-C-C$ group vibrations}

The $\mathrm{C}-\mathrm{C}-\mathrm{C}$ stretching vibrations of cyclododecanone ring predicted at $890-1076 \mathrm{~cm}^{-1}$ correlate well with experimentally observed at 966, 1004, 1013, 1031 and $1080 \mathrm{~cm}^{-1}$. The observed frequency at $490 \mathrm{~cm}^{-1}$ belongs to the $\mathrm{C}-\mathrm{C}-\mathrm{C}$ in-plane bending mode and the appropriate calculated frequency is $486 \mathrm{~cm}^{-1}$ (mode no: 134). In the present work the $\mathrm{C}-\mathrm{C}-\mathrm{C}$ out-of-plane bending mode are calculate at $267 \mathrm{~cm}^{-1}$ (mode no: 148 ).

\subsection{Natural bond orbital analysis}

The NBO analysis has been performed on PAH molecule in order to explain and confirm intramolecular charge transfer (ICT) and the electron density delocalization within the investigated molecule.
The most significant interactions between 'filled' (donor) Lewis type NBO orbitals and 'empty' (acceptor) non Lewis NBO orbitals of PAH molecule are given in Table 2.

In PAH the intramolecular interactions are formed by the orbital overlap between bonding $\mathrm{C}-\mathrm{C}, \mathrm{C}-\mathrm{N}$ and antibonding $\mathrm{C}-\mathrm{C}, \mathrm{C}-\mathrm{N}$ and $\mathrm{C}-\mathrm{O}$ orbitals which results in ICT causing stabilization of the $\mathrm{PAH}$ molecule. The intramolecular hyperconjugative interaction of the $\sigma$ orbital of $\mathrm{C}-\mathrm{C}$ and $\mathrm{C}-\mathrm{N}$ bonds to the antibonding orbital of $\mathrm{C}-\mathrm{C}$ and $\mathrm{C}-\mathrm{N}$ bonds of the glutarimide ring leads to stabilization of the PAH molecule with the stabilization energy in the range of $0.56-2.08 \mathrm{kcal} / \mathrm{mol}$, as evident from Table 2. The intramolecular hyperconjugative interaction of $\sigma(\mathrm{N} 1-\mathrm{C} 2)$ distribute to $\sigma^{*}(\mathrm{~N} 1-\mathrm{C} 6$, $\mathrm{N} 1-\mathrm{C} 21, \mathrm{C} 3-\mathrm{C} 20, \mathrm{C} 6-\mathrm{O} 8$ and $\mathrm{C} 2-09)$ leads to stabilization of $0.56-1.78 \mathrm{kcal} / \mathrm{mol}$. The same kind of interaction is calculated in the distribution from $\sigma(\mathrm{N} 1-\mathrm{C} 6)$, to $\sigma^{*}(\mathrm{~N} 1-\mathrm{C} 2, \mathrm{~N} 1-\mathrm{C} 21$ and $\mathrm{C} 2-\mathrm{O} 9)$ with stabilization energy in the range of $0.97-2.08 \mathrm{kcal} / \mathrm{mol}$. From Table 2 it can be concluded that the bonds in glutarimide ring have electron density of $1.986 \mathrm{e}$, demonstrating strong delocalization in this part of molecule. The highest $\sigma$ electron delocalization originates from $\mathrm{C} 2-\mathrm{C} 3, \mathrm{C} 5-\mathrm{C} 6$ and $\mathrm{N} 1-\mathrm{C} 2$ bonds of the glutarimide ring with $\mathrm{E}(2)$ value in the range $1.16-2.57 \mathrm{kcal} / \mathrm{mol}$. The similar results of $\sigma$ electron delocalization in cyclododecanone ring are found around $\sigma(\mathrm{C}-\mathrm{C})$ bonds of this ring. NBO analysis obviously manifests the evidences of ICT from $\sigma(\mathrm{C} 3-\mathrm{C} 20)$ to $\sigma^{*}(\mathrm{C} 3-\mathrm{C} 7, \mathrm{C} 7-\mathrm{O} 10$, C18-C19 and C19-C20) orbitals with moderate stabilization energy of $0.57-1.33 \mathrm{kcal} / \mathrm{mol}$, Table 2 . Also the results of NBO analysis indicate that strong interaction exists between benzyl group and glutarimide ring. For example, the intramolecular hyperconjugative interaction of $\sigma(\mathrm{N} 1-\mathrm{C} 21)$ distribute to $\sigma^{*}(\mathrm{~N} 1-\mathrm{C} 2, \mathrm{~N} 1-\mathrm{C} 6, \mathrm{C} 2-\mathrm{C} 3$ and $\mathrm{C} 5-\mathrm{C} 6$ ) leading to stabilization of $0.91-2.57 \mathrm{kcal} / \mathrm{mol}$.

The second order perturbation theory analysis of Fock matrix in

Table 2

Second order perturbation theory analysis of Fock matrix in NBO basis.

\begin{tabular}{|c|c|c|c|c|c|c|c|c|}
\hline Donor NBO (i) & Type & $\mathrm{ED}(i)(\mathrm{e})$ & Acceptor NBO (j) & Type & $\operatorname{ED}(j)(e)^{\mathrm{a}}$ & $\mathrm{E}(2)^{\mathrm{b}} \mathrm{kcal} / \mathrm{mol}$ & $\mathrm{E}(j)-\mathrm{E}(i)^{\mathrm{C}}$ a.u. & $\mathrm{F}(i, j)^{\mathrm{d}}$ a.u. \\
\hline N1-C2 & $\sigma$ & 1.98615 & N1-C6 & $\sigma^{*}$ & 0.09366 & 1.12 & 1.21 & 0.034 \\
\hline $\mathrm{N} 1-\mathrm{C} 2$ & $\sigma$ & & N1-C21 & $\sigma^{*}$ & 0.04163 & 1.01 & 1.12 & 0.03 \\
\hline N1-C2 & $\sigma$ & & $\mathrm{C} 2-09$ & $\sigma^{*}$ & 0.01331 & 0.56 & 1.4 & 0.025 \\
\hline $\mathrm{N} 1-\mathrm{C} 2$ & $\sigma$ & & C3-C20 & $\sigma^{*}$ & 0.02287 & 0.75 & 1.17 & 0.027 \\
\hline $\mathrm{N} 1-\mathrm{C} 2$ & $\sigma$ & & C6-08 & $\sigma^{*}$ & 0.01215 & 1.78 & 1.42 & 0.045 \\
\hline N1-C6 & $\sigma$ & 1.98613 & N1-C2 & $\sigma^{*}$ & 0.08661 & 1.16 & 1.21 & 0.034 \\
\hline N1-C6 & $\sigma$ & & N1-C21 & $\sigma^{*}$ & 0.04163 & 0.97 & 1.11 & 0.03 \\
\hline N1-C6 & $\sigma$ & & $\mathrm{C} 2-09$ & $\sigma^{*}$ & 0.01331 & 2.08 & 1.39 & 0.048 \\
\hline C3-C20 & $\sigma$ & 1.96225 & C3-C7 & $\sigma^{*}$ & 0.08676 & 0.95 & 0.93 & 0.027 \\
\hline C3-C2O & $\sigma$ & & C7-010 & $\sigma^{*}$ & 0.01446 & 0.95 & 1.2 & 0.03 \\
\hline C3-C20 & $\sigma$ & & C18-C19 & $\sigma^{*}$ & 0.01391 & 1.33 & 0.99 & 0.033 \\
\hline C3-C2O & $\sigma$ & & $\mathrm{C} 19-\mathrm{C} 20$ & $\sigma^{*}$ & 0.01511 & 0.57 & 0.99 & 0.021 \\
\hline $\mathrm{N} 1-\mathrm{C} 21$ & $\sigma$ & 1.97911 & $\mathrm{~N} 1-\mathrm{C} 2$ & $\sigma^{*}$ & 0.08661 & 1.08 & 1.12 & 0.032 \\
\hline N1-C21 & $\sigma$ & & N1-C6 & $\sigma^{*}$ & 0.09366 & 0.91 & 1.11 & 0.029 \\
\hline N1-C21 & $\sigma$ & & $\mathrm{C} 2-\mathrm{C} 3$ & $\sigma^{*}$ & 0.06963 & 2.57 & 1.08 & 0.048 \\
\hline N1-C21 & $\sigma$ & & C5-C6 & $\sigma^{*}$ & 0.05321 & 2.09 & 1.11 & 0.043 \\
\hline $\mathrm{C} 22-\mathrm{C} 27$ & $\pi$ & 1.65538 & C23-C24 & $\pi^{*}$ & 0.31852 & 20.41 & 0.28 & 0.068 \\
\hline $\mathrm{C} 22-\mathrm{C} 27$ & $\pi$ & & C25-C26 & $\pi^{*}$ & 0.33228 & 20.23 & 0.28 & 0.067 \\
\hline C23-C24 & $\pi$ & 1.65994 & $\mathrm{C} 22-\mathrm{C} 27$ & $\pi^{*}$ & 0.34826 & 20.54 & 0.29 & 0.068 \\
\hline C23-C24 & $\pi$ & & C25-C26 & $\pi^{*}$ & 0.33228 & 20.78 & 0.28 & 0.068 \\
\hline $\mathrm{C} 25-\mathrm{C} 26$ & $\pi$ & 1.66437 & $\mathrm{C} 22-\mathrm{C} 27$ & $\pi^{*}$ & 0.34826 & 20.49 & 0.29 & 0.069 \\
\hline C25-C26 & $\pi$ & & C23-C24 & $\pi^{*}$ & 0.31852 & 19.72 & 0.28 & 0.067 \\
\hline N1 & LP (1) & 1.60913 & C2-09 & $\pi^{*}$ & 0.2328 & 49.67 & 0.27 & 0.109 \\
\hline N1 & $\mathrm{LP}(1)$ & & C6-08 & $\pi^{*}$ & 0.22184 & 46.83 & 0.28 & 0.107 \\
\hline $\mathrm{O} 8$ & LP (2) & 1.86582 & N1-C6 & $\sigma^{*}$ & 0.09366 & 27.84 & 0.66 & 0.122 \\
\hline O8 & $\mathrm{LP}(2)$ & & C5-C6 & $\sigma^{*}$ & 0.05321 & 17.62 & 0.65 & 0.098 \\
\hline O9 & $\mathrm{LP}(2)$ & 1.86776 & N1-C2 & $\sigma^{*}$ & 0.08661 & 26.86 & 0.67 & 0.121 \\
\hline O9 & $\mathrm{LP}(2)$ & & $\mathrm{C} 2-\mathrm{C} 3$ & $\sigma^{*}$ & 0.06963 & 18.77 & 0.63 & 0.099 \\
\hline 010 & $\mathrm{LP}(2)$ & 1.87878 & C3-C7 & $\sigma^{*}$ & 0.08676 & 22.59 & 0.61 & 0.106 \\
\hline 010 & $\mathrm{LP}(2)$ & & C7-C11 & $\sigma^{*}$ & 0.06112 & 19.02 & 0.67 & 0.102 \\
\hline
\end{tabular}

a ED, electron density.

${ }^{b} \mathrm{E}(2)$ means energy of hyperconjugative interactions.

c Energy difference between donor and acceptor $i$ and $j$ NBO orbitals.

${ }^{\mathrm{d}} \mathrm{F}(i, j)$ is the Fock matrix element between $i$ and $j$ NBO orbitals. 
Table 3

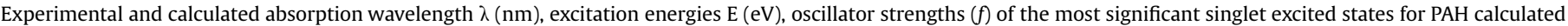
with B3LYP/6-311++G(d,p) method.

\begin{tabular}{|c|c|c|c|c|}
\hline \multirow[t]{2}{*}{ Transition } & \multirow[t]{2}{*}{ Experimental $\lambda(\mathrm{nm})$} & \multicolumn{3}{|c|}{$\mathrm{TD}(\mathrm{DFT})$ method $\mathrm{CHCl}_{3}$} \\
\hline & & $\lambda(\mathrm{nm})$ & Excitation energy $\mathrm{E}(\mathrm{eV})$ & Oscillator strength $f$ \\
\hline I & 340 & 291.05 & $5.2599(98 \rightarrow 101)$ & 0.0061 \\
\hline II & 285 & 239.57 & $5.1752(100 \rightarrow 102)$ & 0.01 \\
\hline III & & 234.07 & $5.2969(96 \rightarrow 101)$ & 0.0227 \\
\hline
\end{tabular}

NBO basis of PAH (Table 2) also points out intramolecular interaction due to the orbital overlap of $\pi(\mathrm{C}-\mathrm{C})$ and $\pi^{*}(\mathrm{C}-\mathrm{C})$ of phenyl group, resulting in high electron density (approx. $0.335 \mathrm{e}$ ) of antibonding $\pi$ orbitals $(\mathrm{C}-\mathrm{C})$.

The interaction energy, related to the resonance in the molecule, is electron withdrawing to the ring through $\sigma^{*}$ of N1-C6 and C5-C6 bonds from the lone pair $\mathrm{LP}(2) \mathrm{O} 8$ which leads to stabilization energy of 27.84 and $17.62 \mathrm{kcal} / \mathrm{mol}$, respectively. Also, the magnitude of charge transfer from the lone pairs of $\mathrm{LP}(2) \mathrm{O}$ atom to antibonding $\sigma$ orbitals $\mathrm{C} 2-\mathrm{C} 3$ and $\mathrm{N} 1-\mathrm{C} 2$ of glutarimide ring, amount to stabilization of 18.77 and $26.86 \mathrm{kcal} / \mathrm{mol}$, while from LP(2)O10 to anti-bonding $\sigma$ orbitals C7-C11 and C3-C7 of cyclododecanone ring is of 19.02 and $22.59 \mathrm{kcal} / \mathrm{mol}$, respectively. The interaction energies of $\mathrm{LP}(1) \mathrm{N} 1 \rightarrow \pi^{*}(\mathrm{C} 2-\mathrm{O} 9)$ and $\mathrm{LP}(1) \mathrm{N} 1 \rightarrow$ $\pi^{*}(\mathrm{C} 6-08)$ are 49.67 and $46.83 \mathrm{kcal} / \mathrm{mol}$, respectively. These results additionally confirm the existence of ICT in the investigated molecule.

\subsection{Electronic properties}

The electronic absorption spectrum of the title compound in chloroform solvent was recorded within the $200-500 \mathrm{~nm}$ range and the representative spectrum of computed transitions plot on the experimental is shown in Fig. S2 (Supplementary material). The electronic absorption spectrum of PAH was calculated by the TDDFT on the B3LYP/6-311++G(d,p) level. The solvent effect was calculated with CPCM method [31]. The calculated vertical excitation energies, wavelength $(\lambda)$, oscillator strength $(f)$, composition of the most significant singlet excited states and experimentally obtained wavelengths are shown in Table 3.

The structure of PAH allow $\sigma-\sigma^{*}$ and $\pi-\pi^{*}$ transition in the UVVis spectrum. NBO analysis indicates that most of molecular orbitals are $\sigma$ type orbitals, so the electronic transitions are mainly derived from the contribution of $\sigma-\sigma^{*}$ bands. However, NBO analysis shows that the electronic transitions derived from $\pi-\pi^{*}$ orbitals are dominant and stronger (higher E(2) values, Table 2). The TD-DFT calculation predicts one low intense electronic transition at $291.05 \mathrm{~nm}$ with an oscillator strength $f=0.0061$ which is in good agreement with the experimentally obtained maximum with $\lambda_{\exp }=340 \mathrm{~nm}$ in chloroform, Fig. S2 (Supplementary material). The first transition is between HOMO-2 (orbital 98) and LUMO (orbital 101). The next transition is HOMO (orbital 100) to LUMO+1 (orbital 102) at $239.57 \mathrm{~nm}$, with oscillation strength of 0.01 which correlates well with experimentally found peak at $285 \mathrm{~nm}$, Table 3.

The frontier orbitals, HOMO and LUMO illustrate the way the molecules interact with other species. Therefore, FMO analysis helps to elucidate the chemical reactivity of molecules.

The lowest unoccupied molecular orbital is localized mainly on glutarimide ring and on the part $\mathrm{C} 20-\mathrm{C} 3-\mathrm{C} 11$ of cyclododecanone ring as shown in Fig. 7. The highest occupied molecular orbital is spread over the benzyl group, imide group of glutarimide ring and the carbonyl group $C 7=010$. The HOMO (orbital 100) to LUMO (orbital 101) transition implies an electron density transfer to the cyclododecanone ring from the benzyl and imide groups $\left(\pi \rightarrow \pi^{*}\right.$ transition) and from the lone pair on nitrogen and oxygen atoms ( $\mathrm{n}$ $\rightarrow \pi^{*}$ transition). The HOMO laying at $-6.97311 \mathrm{eV}$ and the LUMO laying at $-1.45873 \mathrm{eV}$ (computed by TD-DFT) and the energy gap is $5.51 \mathrm{eV}$.

\subsection{NMR analysis}

It is well known that chemical shifts of hydrogen and carbon atoms in NMR spectra contain information about the molecular structure. Also, the chemical shifts show sensitivity according to conformational variations. The combination of experimental and computational NMR spectra is used to prove the structure of the most stable isomer of PAH molecule. The experimental NMR
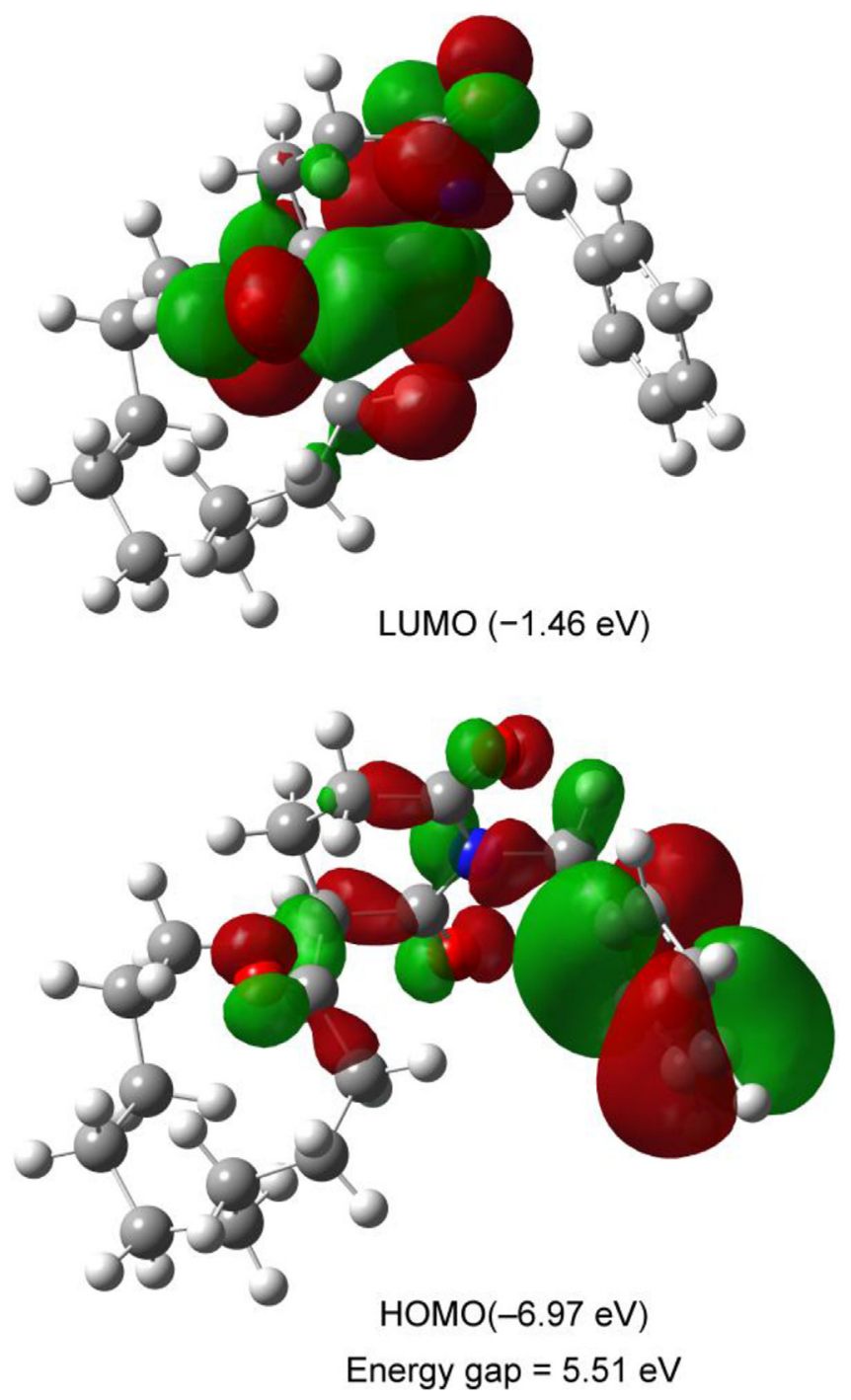

Fig. 7. The frontier molecular orbitals (HOMO and LUMO) for PAH. 
Table 4

Theoretical and experimental ${ }^{1} \mathrm{H}$ NMR chemical shifts of $\mathrm{PAH}$ compound in $\mathrm{CDCl}_{3}$ solution (atom positions were numbered as in Fig. 2).

\begin{tabular}{|c|c|c|}
\hline Hydrogen & B3LYP/6311++G(d,p) & Experimental \\
\hline $28 \mathrm{H}$ & 1.94 & 1.97 \\
\hline $29 \mathrm{H}$ & 0.90 & 0.91 \\
\hline $30 \mathrm{H}$ & 3.18 & 3.19 \\
\hline $31 \mathrm{H}$ & 1.04 & 1.19 \\
\hline $32 \mathrm{H}$ & 1.11 & 1.33 \\
\hline $33 \mathrm{H}$ & 1.20 & 1.51 \\
\hline $34 \mathrm{H}$ & 1.04 & 1.19 \\
\hline $35 \mathrm{H}$ & 1.38 & 1.58 \\
\hline $36 \mathrm{H}$ & 1.28 & 1.52 \\
\hline $37 \mathrm{H}$ & 1.36 & 1.56 \\
\hline $38 \mathrm{H}$ & 1.16 & 1.31 \\
\hline $39 \mathrm{H}$ & 1.20 & 1.50 \\
\hline $40 \mathrm{H}$ & 1.31 & 1.53 \\
\hline $41 \mathrm{H}$ & 1.34 & 1.54 \\
\hline $42 \mathrm{H}$ & 1.37 & 1.57 \\
\hline $43 \mathrm{H}$ & 1.28 & 1.53 \\
\hline $44 \mathrm{H}$ & 1.13 & 1.50 \\
\hline $45 \mathrm{H}$ & 0.94 & 0.94 \\
\hline $46 \mathrm{H}$ & 2.58 & 1.59 \\
\hline $47 \mathrm{H}$ & 1.32 & 1.54 \\
\hline $48 \mathrm{H}$ & 1.52 & 2.59 \\
\hline $49 \mathrm{H}$ & 2.53 & 2.56 \\
\hline $50 \mathrm{H}$ & 2.67 & 2.69 \\
\hline $51 \mathrm{H}$ & 2.66 & 2.67 \\
\hline $52 \mathrm{H}$ & 5.52 & 5.01 \\
\hline $53 \mathrm{H}$ & 4.50 & 4.91 \\
\hline $54 \mathrm{H}$ & 7.72 & 7.34 \\
\hline $55 \mathrm{H}$ & 7.43 & 7.22 \\
\hline $56 \mathrm{H}$ & 7.57 & 7.27 \\
\hline $57 \mathrm{H}$ & 7.57 & 7.22 \\
\hline $58 \mathrm{H}$ & 7.82 & 7.34 \\
\hline
\end{tabular}

spectra of PAH were recorded in $\mathrm{CDCl}_{3}$ and shown in Figs. S3 and S4 (Supplementary material). The experimental and theoretical values for ${ }^{1} \mathrm{H}$ and ${ }^{13} \mathrm{C}$ NMR shifts of PAH are presented in Tables 4 and 5.

In experimental ${ }^{1} \mathrm{H}$ NMR spectrum of $\mathrm{PAH}$, shifts of aliphatic $\mathrm{C}-\mathrm{H}$ protons are occurred in the range of $0.91-5.91 \mathrm{ppm}$ and the calculated are in the range $0.90-5.52 \mathrm{ppm}$. There are many differences in the chemical shifts in the aliphatic region of this spectrum. For example, the chemical shift of hydrogen attached to carbon near the carbonyl group is higher than one that would be expected regarding the electron density around the hydrogen. The hydrogen atom of $\mathrm{C} 11-\mathrm{H} 30$ group appears at higher chemical shift of $3.19 \mathrm{ppm}$ due to influence of carbonyl group $\mathrm{C} 7=\mathrm{O} 10$ with theoretical peak at $3.18 \mathrm{ppm}$. The same applies for the hydrogens bonded to $\mathrm{C} 20$ (H46 and H47) appear at 1.59 and 1.54 ppm due to influence of carbonyl group $\mathrm{C} 2=09$. Two multiple signals appearing at 2.56, 2.59 and 2.67, $2.69 \mathrm{ppm}$ are assigned to protons ( $\mathrm{H} 49, \mathrm{H} 48$ and $\mathrm{H} 51, \mathrm{H} 50$ ) of two methylene groups in glutarimide ring, $\mathrm{C}_{4} \mathrm{H}_{2}$ and $\mathrm{C}_{5} \mathrm{H}_{2}$ of $\mathrm{PAH}$ (Table 4). These signals show good agreement with theoretical values in the region $1.52-2.67 \mathrm{ppm}$. Two doublets of doublets at 4.91 and $5.01 \mathrm{ppm}$ (in $\mathrm{CDCl}_{3}$ solution) mark the methylene hydrogens $\mathrm{H} 53$ and $\mathrm{H} 52$ of methylene group $\mathrm{C}_{2} 1 \mathrm{H}_{2}$. The calculated chemical shift values for these hydrogen atoms (with respect to TMS) are 4.5 and $5.52 \mathrm{ppm}$ in $\mathrm{CDCl}_{3}$ solution. The calculated chemical shift values for phenyl ring hydrogens are at 7.43-7.82 ppm and experimental peaks for these atoms are in the range of $7.22-7.34 \mathrm{ppm}$.

Chemical shifts of the carbon atoms in ${ }^{13} \mathrm{C}$ NMR spectra of $\mathrm{PAH}$ do not show unexpected values. In the experimental ${ }^{13} \mathrm{C}$ NMR spectrum, carbon atoms of methylene groups of cyclododecanone ring show signals in the range of $18.78-34.66 \mathrm{ppm}$ and correlate very well with the computed values in the range of 22.8-40.29 ppm, Table 5. The methylene carbon atoms of glutarimide ring C4 and C5 appear at 23.26 and 29.94 ppm, and
Table 5

Theoretical and experimental ${ }^{13} \mathrm{C}$ NMR chemical shifts of PAH compound in $\mathrm{CDCl}_{3}$ solution (atom positions were numbered as in Fig. 2).

\begin{tabular}{lll}
\hline Carbon & B3LYP/6-311++G(d,p) & Experimental \\
\hline 2 C & 182.53 & 171.9 \\
3 C & 68.24 & 59.96 \\
4 C & 28.82 & 23.26 \\
5 C & 35.71 & 29.94 \\
6 C & 183.35 & 172.68 \\
7 C & 219.09 & 204.81 \\
$11 \mathrm{C}$ & 40.29 & 34.66 \\
$12 \mathrm{C}$ & 26.33 & 21.99 \\
$13 \mathrm{C}$ & 27.01 & 23.07 \\
$14 \mathrm{C}$ & 26.63 & 22.4 \\
$15 \mathrm{C}$ & 31.93 & 26.26 \\
$16 \mathrm{C}$ & 26.19 & 21.66 \\
$17 \mathrm{C}$ & 24.93 & 21.21 \\
$18 \mathrm{C}$ & 31.41 & 26.21 \\
$19 \mathrm{C}$ & 22.80 & 18.78 \\
$20 \mathrm{C}$ & 39.14 & 33.81 \\
$21 \mathrm{C}$ & 48.33 & 43.39 \\
$22 \mathrm{C}$ & 146.99 & 136.92 \\
$23 \mathrm{C}$ & 136.45 & 128.81 \\
$24 \mathrm{C}$ & 135.48 & 128.4 \\
$25 \mathrm{C}$ & 134.10 & 127.52 \\
$26 \mathrm{C}$ & 134.86 & 128.4 \\
$27 \mathrm{C}$ & 138.54 & 128.81 \\
\hline
\end{tabular}

calculated values are 28.82 and $35.71 \mathrm{ppm}$. Spiro carbon atom C3 is observed at $59.96 \mathrm{ppm}$, whereas the corresponding computed value is $68.24 \mathrm{ppm}$. Signals for aromatic carbons are observed in the range 127.52-136.92 ppm for the phenyl ring of PAH. The carbonyl atoms C2, C6 and C7 resonating at 171.9, 172.68 and $204.81 \mathrm{ppm}$ and computed values are $182.53,183.35$ and $219.09 \mathrm{ppm}$.

\subsection{Molecular electrostatic potential (MEP)}

Considering the great anticancer activity of PAH molecule, it was necessary to find the molecular fragments that are responsible for this activity as well as for chemical reactivity. The analysis of molecular electrostatic potential could predict those parts of molecule responsible for the electrophilic and nucleophilic reactions as well as inter- and/or intra-molecular bonds. The MEP at the B3LYP/6$311++\mathrm{G}(\mathrm{d}, \mathrm{p})$ optimized geometry was calculated and depicted in Fig. 8. The negative regions of the MEP are related to electrophilic reactivity and are colored in red and yellow. The positive, blue regions are related to nucleophilic reactivity, Fig. 8. As can be seen from the figure, negative region is localized over the $\mathrm{N}$ and $\mathrm{O}$ atoms. The maximum positive region, dark blue, is localized on the $\mathrm{C}-\mathrm{H}$

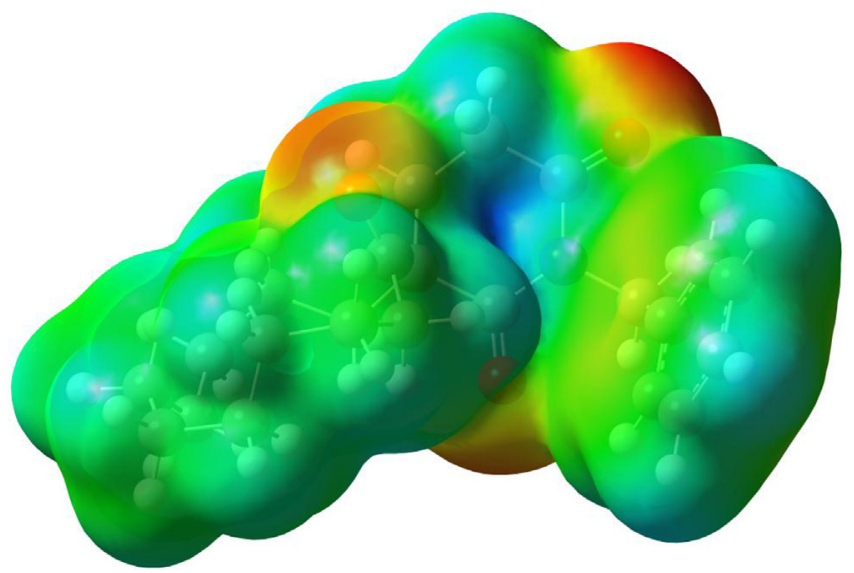

Fig. 8. MEP of investigated molecule calculated with B3LYP/6-311++G(d,p) method. 
bonds of glutarimide ring. This region gives information about possible sites for nucleophilic attack. According to these calculated results, the MEP map shows that the negative sites are on electronegative nitrogen atom of the imide group and oxygen atoms of carbonyl groups. The positive sites are around the hydrogen atoms of glutarimide ring.

\section{Conclusion}

The conformational stability of PAH is thoroughly studied and results are presented in this paper. The most stable conformer of the glutarimide spiro derivative PAH was found and its spectroscopic properties were investigated experimentally and with the help of DFT. The most stable conformer I has cis orientation of benzyl group and carbonyl group of cyclododecanone ring. The conformational, vibrational, electronic and NMR analyses were done with B3LYP/6-311++G(d,p) method. In addition, NBO analysis has provided evidence of the ICT through the molecule. The MEP map anticipates that imide fragment (oxygen and nitrogen atoms) of $\mathrm{PAH}$ is the most reactive site for electrophilic and hydrogen atoms of methylene groups of glutarimide ring for nucleophilic attack.

\section{Acknowledgments}

This work has been financially supported by Ministry of Education and Science, Republic of Serbia, under Grant Nos. 172035 and 172032. Numerical simulations were run on the PARADOX cluster at the Scientific Computing Laboratory of the Institute of Physics Belgrade, supported in part by the Ministry of Education, Science and Technological Development of the Republic of Serbia under project No. ON171017.

\section{Appendix A. Supplementary data}

Supplementary data associated with this article can be found in the online version, at http://dx.doi.org/10.1016/j.molstruc.2017.02. 012. These data include MOL files and InChiKeys of the most important compounds described in this article.

\section{References}

[1] F. Matsuda, S. Terashima, Tetrahedron 44 (1988) 4721-4736, http:// dx.doi.org/10.1016/S0040-4020(01)86175-5.

[2] J. Ju, S.R. Rajski, S.-K. Lim, J.-W. Seo, N.R. Peters, F.M. Hoffmann, B. Shen, J. Am. Chem. Soc. 131 (2009) 1370-1371, http://dx.doi.org/10.1021/ja808462p.

[3] K. Sugawara, Y. Nishiyama, S. Toda, N. Komiyama, M. Hatori, T. Moriyama, Y. Sawada, H. Kamei, M. Konishi, T. Oki, J. Antibiot. 45 (1992) 1433-1441, http://dx.doi.org/10.7164/antibiotics.45.1433.

[4] A.L. Machado, L.M. Lima, J.X. Araujo Jr., C.A.M. Fraga, V.L.G. Koatz, E.J. Barreiro, Bioorg. Med. Chem. Lett. 15 (2005) 1169-1172, http://dx.doi.org/10.1016/ j.bmcl.2004.12.012.

[5] J.B. Bartlett, K. Dredge, A.G. Dalgleish, Nat. Rev. Cancer 4 (2004) 314-322, http://dx.doi.org/10.1038/nrc1323.

[6] Y.-H. Wu, J.W. Rayburn, L.E. Allen, H.C. Ferguson, J.W. Kissel, J. Med. Chem. 15 (1972) 477-479, http://dx.doi.org/10.1021/jm00275a009.

[7] L.B. Barradell, A. Fitton, CNS Drugs 5 (1996) 147-152, http://dx.doi.org/ 10.2165/00023210-199605020-00006.

[8] T.G. Obrig, W.J. Culp, W.L. McKeehan, B. Hardesty, J. Biol. Chem. 246 (1971) 174-181.

[9] H. Kondo, T. Oritani, H. Kiyota, Eur. J. Org. Chem. 2000 (2000) 3459-3462, http://dx.doi.org/10.1002/1099-0690(200010)2000:20<3459::AIDEJOC3459>3.0.CO;2-E.

[10] R.P. Frohardt, H.W. Dion, Z.L. Jakubowski, A. Rydeer, J.C. French, Q.R. Bartz,
J. Am. Chem. Soc. 81 (1959) 5500-5506, http://dx.doi.org/10.1021/ ja01529a059.

[11] B.S. Kim, S.S. Moon, B.K. Hwang, J. Agric. Food Chem. 47 (1999) 3372-3380, http://dx.doi.org/10.1021/jf981259s.

[12] D.K.K. Ha, W.H. Lau, Cancer Lett. 41 (1988) 217-224, http://dx.doi.org/ 10.1016/0304-3835(88)90119-X.

[13] M.I. Andres, P. Sanz, A. Garfia, G. Repetto, M. Repetto, In Vitro Toxicol. 10 (1997) 319-328.

[14] X. Armoiry, G. Aulagner, T. Facon, J. Clin. Pharm. Ther. 33 (2008) 219-226, http://dx.doi.org/10.1111/j.1365-2710.2008.00920.x.

[15] S. Lentzsch, M.S. Rogers, R. LeBlanc, A.E. Birsner, J.H. Shah, A.M. Treston, K.C. Anderson, R.J. D'Amato, Cancer Res. 62 (2002) 2300-2305.

[16] I. Antonini, R. Volpini, D. Dal Ben, C. Lambertucci, G. Cristalli, Bioorgan. Med. Chem. 16 (2008) 8440-8446, http://dx.doi.org/10.1016/j.bmc.2008.08.027.

[17] J.T. Norton, M.A. Witschi, L. Luong, A. Kawamura, S. Ghosh, M.S. Stack, E. Sim M.J. Avram, D.H. Appella, S. Huang, Anti Cancer Drugs 19 (2008) 23-26, http:/ dx.doi.org/10.1097/CAD.0b013e3282f00e17.

[18] A. Wu, Y. Xu, X. Qian, J. Wang, J. Liu, Eur. J. Med. Chem. 44 (2009) 4674-4680, http://dx.doi.org/10.1016/j.ejmech.2009.07.011.

[19] M.K. Hargreaves, J.G. Pritchard, H.R. Dave, Chem. Rev. 70 (1970) 439-469, http://dx.doi.org/10.1021/cr60266a001.

[20] J.B. Popović-Djordjević, A.S. Klaus, Ž.S. Žižak, I.Z. Matić, B.J. Drakulić, J. Enzyme Inhib. Med. Chem. 31 (2016) 915-923, http://dx.doi.org/10.3109/ 14756366.2015.1070844.

[21] J.B. Popović-Đorđević, M.D. Ivanović, V.D. Kiricojević, Tetrahedron Lett. 46 (2005) 2611-2614, http://dx.doi.org/10.1016/j.tetlet.2005.02.087.

[22] R.W. Harrison, J. Comput. Chem. 14 (1993) 1112-1122, http://dx.doi.org/ $10.1002 /$ jcc.540140911.

[23] A. Pedretti, L. Villa, G. Vistoli, J. Comput. Aid Mol. Des. 18 (2004) 167-173, http://dx.doi.org/10.1023/B: JCAM.0000035186.90683.f2.

[24] W. Humphrey, A. Dalke, K. Schulten, J. Mol. Graph 14 (1996) 33-38, http:// dx.doi.org/10.1016/0263-7855(96)00018-5.

[25] M.J. Frisch, G.W. Trucks, H.B. Schlegel, G.E. Scuseria, M.A. Robb, J.R. Cheeseman, G. Scalmani, V. Barone, B. Mennucci, G.A. Petersson, H. Nakatsuji, M. Caricato, X. Li, H.P. Hratchian, A.F. Izmaylov, J. Bloino, G. Zheng, J.L. Sonnenberg, M. Hada, M. Ehara, K. Toyota, R. Fukuda, J. Hasegawa, M. Ishida, T. Nakajima, Y. Honda, O. Kitao, H. Nakai, T. Vreven, J.A. Montgomery Jr., J.E. Peralta, F. Ogliaro, M. Bearpark, J.J. Heyd, E. Brothers, K.N. Kudin, V.N. Staroverov, R. Kobayashi, J. Normand, K. Raghavachari, A. Rendell, J.C. Burant, S.S. Iyengar, J. Tomasi, M. Cossi, N. Rega, J.M. Millam, M. Klene, J.E. Knox, J.B. Cross, V. Bakken, C. Adamo, J. Jaramillo, R. Gomperts, R.E. Stratmann, O. Yazyev, A.J. Austin, R. Cammi, C. Pomelli, J.W. Ochterski, R.L. Martin, K. Morokuma, V.G. Zakrzewski, G.A. Voth, P. Salvador, J.J. Dannenberg, S. Dapprich, A.D. Daniels, Ö. Farkas, J.B. Foresman, J.V. Ortiz, J. Cioslowski, D.J. Fox, Gaussian 09, Revision D.01, Gaussian Inc., Wallingford, CT, 2009.

[26] M.H. Jamróz, Vibrational Energy Distribution Analysis VEDA 4, 2004. Warsaw.

[27] A.P. Scott, L. Radom, J. Phys. Chem. 100 (1996) 16502-16513, http:// dx.doi.org/10.1021/jp960976r.

[28] B.J. Orr, J.F. Ward, Mol. Phys. 20 (1970) 513-526, http://dx.doi.org/10.1080/ 00268977000101521.

[29] R. Ditchfield, J. Chem. Phys. 56 (1972) 5688-5691, http://dx.doi.org/10.1063/ 1.1677088.

[30] K. Wolinski, J.F. Hinton, P. Pulay, J. Am. Chem. Soc. 112 (1990) 8251-8260, http://dx.doi.org/10.1021/ja00179a005.

[31] M. Cossi, N. Rega, G. Scalmani, V. Barone, J. Comput. Chem. 24 (2003) 669-681, http://dx.doi.org/10.1002/jcc.10189.

[32] E.D. Glendening, A.E. Reed, J.E. Carpenter, F. Weinhold, NBO version 3.1, TCI, University of Wisconsin, Madison, 1998.

[33] V.M. Arsovski, B.Đ. Božić, J.M. Mirković, V.D. Vitnik, Ž.J. Vitnik, S.D. Petrović, G.S. Ušćumlić, D.Ż. Mijin, J. Mol. Mod. 21 (2015) 239, http://dx.doi.org/ 10.1007/s00894-015-2777-z.

[34] V.D. Vitnik, Ž.J. Vitnik, Spectrochim. Acta A 138 (2015) 1-12, http://dx.doi.org/ 10.1016/j.saa.2014.11.005.

[35] V.D. Vitnik, Ž.J. Vitnik, N.R. Banjac, N.V. Valentić, G.S. Ušćumlić, I.O. Juranić, Spectrochim. Acta A 117 (2014) 42-53, http://dx.doi.org/10.1016/ j.saa.2013.07.099.

[36] V.A. Minaeva, B.F. Minaeva, G.V. Baryshnikov, H. Ågren, M. Pittelkow, Vib. Spectrosc. 61 (2012) 156-166, http://dx.doi.org/10.1016/j.vibspec.2012.02.005.

[37] T.A. Rudol'fi, A.A. Skorubskii, É.B. Krymskaya, J. Appl. Spectrosc. 22 (1975) 1001-1003, http://dx.doi.org/10.1007/BF00608833.

[38] P. Dowd, S.-C. Choi, Tetrahedron 48 (1992) 4773-4792, http://dx.doi.org/ 10.1016/S0040-4020(01)81574-X.

[39] M. Beer, H.B. Kessler, G.B.B.M. Sutherland, J. Chem. Phys. 29 (1958) 1097-1104, http://dx.doi.org/10.1063/1.1744662. 